\title{
Diffusion barriers at Mars surface conditions: Salt crusts, particle size mixtures, and dust
}

\author{
Troy L. Hudson ${ }^{1}$ and Oded Aharonson ${ }^{1}$ \\ Received 22 October 2007; revised 27 February 2008; accepted 4 June 2008; published 18 September 2008.
}

[1] The diffusion coefficient of water vapor through porous media at Mars-like surface conditions is measured for a variety of complex particle size distributions and soil compositions. Micron-sized dust simulants, mixtures of sand- and dust-sized particles, and salt-encrusted sand are examined. We find that while the value of the diffusion coefficient, $D$, can be reduced by up to a factor of 10 for heavily salt-encrusted soils (minimum observed $D=0.4 \pm 0.04 \mathrm{~cm}^{2} \mathrm{~s}^{-1}$ ), moderate amounts of salt only produce minor reductions in $D$. Mechanical packing of pure dust can lower $D$ by a similar amount, while mixtures of dust with sand-sized particles produce at most a factor of $\sim 4$ reduction. We conclude that present-day processes of aeolian redistribution, moderate levels of salt encrustation, and volatile loss from dirty ice would be inefficient at producing soil deposits and lags on Mars that pose significant barriers to diffusion. Therefore, subsurface ice deposits that are thermally unstable would not be protected against sublimative loss by such materials.

Citation: Hudson, T. L., and O. Aharonson (2008), Diffusion barriers at Mars surface conditions: Salt crusts, particle size mixtures, and dust, J. Geophys. Res., 113, E09008, doi:10.1029/2007JE003026.

\section{Introduction}

[2] Subsurface ice on Mars is extensive at present, having been observed within a meter of the surface at latitudes poleward of $\sim 60^{\circ}$ [Boynton et al., 2002]. Past epochs on Mars no doubt also harbored buried ice, though the distribution of the ice may have been significantly different [Mellon and Jakosky, 1993, 1995; Carr, 1996]. A number of investigations have examined the equilibrium behavior of subsurface ice under the present climate [e.g., Mellon and Jakosky, 1993; Schorghofer and Aharonson, 2005] and under various climate conditions as modulated by orbital parameters [Mellon and Jakosky, 1995; Mellon et al., 1997; Levrard et al., 2005]. The ice, though protected from extremes of temperature by the overlying regolith, is still in diffusive contact with the atmosphere and is affected by parameters such as vapor content and insolation. While not directly related to the equilibrium position of the ice table, the diffusive properties of the porous media overlying ice exert a first-order control on the rate of response of the ice table's position to changing conditions.

[3] As reviewed by Hudson et al. [2007], previous investigations of diffusion on Mars have used various estimates or extrapolations for the value of the diffusion coefficient, empirical data on diffusivity of porous material at Mars surface conditions being unavailable. Many of the estimates used were based on experiments in uniform granular media at Earth-ambient temperatures and pres-

\footnotetext{
${ }^{1}$ Division of Geological and Planetary Sciences, California Institute of Technology, Pasadena, California, USA.

Copyright 2008 by the American Geophysical Union. 0148-0227/08/2007JE003026\$09.00
}

sures. However, as demonstrated by all landed Mars missions, the surface of Mars exhibits a wide variety of soil types with different particle sizes, morphologies, and compositions [Yen et al., 2005] and small regions exhibit heterogeneous distribution of these types. This suggests that the diffusive properties of the Martian regolith may vary over small length scales. Such local variations may comprise large regional areas which exhibit a generally larger or smaller diffusive potential than the global average, and thus strongly influence the global Mars water cycle.

[4] We have previously published experiments which determine the diffusive properties of water vapor through unconsolidated regolith materials in $\mathrm{CO}_{2}$ carrier gas under Mars-like surface conditions of temperature, pressure, and humidity [Hudson et al., 2007]. Those results show that a wide variety of homogeneous surface materials exhibit largely similar diffusion coefficients from $\sim 2$ to $5 \mathrm{~cm}^{2}$ $\mathrm{s}^{-1}$ at $200 \mathrm{~K}$. The effects on diffusivity of more complicated regoliths, which may arise from Mars surface processes, are the subject of the subsequent investigations here. In this work, we consider the results of two generalized processes which may act to reduce the rate of communication between the atmosphere and the subsurface: the mixing of dust into deposits of sand-sized particles and the formation of saltcemented crusts. Mixing may arise from impact comminution and gardening of the surface, aeolian reworking, or any other process which will allow the ubiquitous Martian dust access to the internal pore spaces of coarser soil. Salt encrustation as envisioned here requires the presence of water as a solvent, though the amount of liquid required and the role of vapor on Mars is unknown. The discovery of subsurface soils with high salt contents $(>30 \%)$ at the Mars Exploration Rover sites and additional evidence that argues 
for the presence of stable surface water at some point in the early history of Mars encourages investigation of past processes. The detection of surface duricrusts at all landing sites suggests that more recent (and possibly ongoing) action of surface frost and thin films of meltwater could currently operate to redistribute salt.

[5] Our experiments on salt crusts and mixtures of sand and dust are reported below. Section 2 gives a brief overview of the diffusion theory used in interpreting the results. Sections 3 and 4 detail the experimental materials and methods, respectively. Section 5 describes the results of more than 75 separate experiments. The implications of the results of these studies to subsurface ice systems are covered in section 6 .

\section{Diffusion Theory}

[6] The evolution of ice in diffusive contact with a planet's atmosphere through a barrier of porous material is affected by the thermal and geometric properties of the regolith. The former controls the propagation of diurnal, annual, and long-term thermal waves into the subsurface and thereby modulates the time-averaged temperatures experienced at depth. The latter set of properties, including porosity, pore size and shape, and tortuosity, influence the rate at which gas molecules migrate in response to chemical potential gradients. Temperature, through its effect on the saturation vapor density of air and the vapor pressure of ice, determines the concentration of water molecules in the subsurface. Changing temperatures may create a positive, negative, or identically zero concentration gradient with respect to the vapor density in the atmosphere. If a gradient of concentration exists, there will be a net flux of water molecules down the gradient, resulting in a net growth or depletion of the subsurface ice with time. The magnitude of this flux depends both on the magnitude of the concentration gradient and on the diffusive properties of the soil.

[7] A description of the theoretical framework for diffusive vapor transport in a sublimation environment on Mars is found by Hudson et al. [2007]. An abbreviated summary is given here. Throughout this paper, subscript 1 refers to $\mathrm{H}_{2} \mathrm{O}$ and subscript 2 refers to the carrier gas. The quantites of pressure and mass are denoted by $p_{0}$, and $\rho_{0}$, respectively. The free gas diffusion coefficient is denoted by $\mathcal{D}_{12}$, while the diffusivity in a porous medium is referred to by $D$. The mass flux of water vapor, ignoring thermodiffusion and barodiffusion [Landau and Lifshitz, 1987] but including advection, is

$$
J=-\rho_{0} D \frac{\partial}{\partial z} \frac{\rho_{1}}{\rho_{0}}-\rho_{1} \frac{\kappa}{\mu} \frac{\partial p_{0}}{\partial z}
$$

where $z$ is depth, $\kappa$ is the intrinsic permeability of the porous medium, and $\mu$ is the dynamic viscosity of the gas. The first term on the right is the diffusive mass flux $J_{\text {diff, }}$ and the second term is the advective flux $J_{\text {adv }}$.

[8] When ice is lost from an impermeable ice layer, the ratio of advective to diffusive flux is related to the concentration of water vapor, $c=\rho_{1} / \rho_{0}$, by [Hudson et al., 2007]

$$
\frac{J_{\mathrm{adv}}}{J_{\mathrm{diff}}}=\frac{c}{1-c} .
$$

In our previous experiments at $263 \mathrm{~K}$ [Hudson et al., 2007], it was determined that $\sim 10 \%$ of the total flux through the sample was due to advection. In the more recent experiments carried out at $250 \mathrm{~K}$ and described in section 5.1 , the advective contribution is $\sim 3 \%$. There is always some component of the flux which is advective, but by using a colder environment we are able to reduce the systematic error in our flux measurement by about a factor of 3 .

[9] Variations of $\rho_{0}$ with depth are small in our experimental setup and will be neglected. An approximate expression for concentration-driven diffusion in a porous medium is thus

$$
J=-D \frac{\partial \rho_{1}}{\partial z}
$$

[10] In a porous medium, the diffusion coefficient is reduced relative to diffusion in a free gas by an obstruction factor often represented by the quotient of the porosity, $\phi$, and the tortuosity, $\tau$ [Mason and Malinauskas, 1983]:

$$
D=\frac{\phi}{\tau} \bar{D}
$$

where $D$ is the effective diffusivity of the medium and $\bar{D}$ is termed the reference diffusivity. Here we follow the method of Zalc et al. [2004], wherein a strictly geometric tortuosity factor, independent of diffusion regime, is derived. In this formalism, the Bosanquet equation gives the reference diffusivity as

$$
\bar{D}=\left(\frac{1}{\mathcal{D}_{12}}+\frac{1}{D_{K}}\right)^{-1}
$$

where $\mathcal{D}_{12}$ and $D_{K}$ are the bulk or free gas diffusivity and the Knudsen diffusivity, respectively. Porosity quantifies the reduction in cross-sectional area available for gaseous transport, while tortuosity is a quantity which characterizes the convoluted nature of the porous pathways followed by diffusing species. The theoretical determination of tortuosity is model dependent and extremely cumbersome for all but the most simple geometries. It is most often the case that the other parameters in equation (3), $D, \phi$, and $\bar{D}$, are determined from experiment and that $\tau$ is calculated from these. The majority of experiments by Hudson et al. [2007] were shown to be at the Fickian edge of the transition region, and the experiments reported here occur at the same pressures $(\sim 600 \mathrm{~Pa})$. Thus, the diffusion coefficients reported are the effective diffusion coefficients, $D$.

[11] The diffusion of water molecules in $\mathrm{CO}_{2}$ and $\mathcal{D}_{12}$ may be derived via Chapman-Enskog theory and by a variety of extrapolations from measured data to the appropriate temperatures and pressures. Under Fickian diffusion or in free gas, where collisions between molecules are more frequent than collisions with pore walls, the diffusion coefficient is proportional to $p_{0}^{-1}$ and, when a molecular model of hard elastic spheres is assumed, to $T^{3 / 2}$ [Chapman and Cowling, 1970]. For instance, Wallace and Sagan [1979], write $\mathcal{D}_{\mathrm{H}_{2} \mathrm{O} \cdot \mathrm{CO}_{2}}=\left(0.1654 \mathrm{~cm}^{2} \mathrm{~s}^{-1}\right)\left(T / T_{\text {ref }}\right)^{3 / 2}\left(p_{\text {ref }}\right.$ $p_{0}$ ), where $p_{\text {ref }}=1013 \mathrm{mbar}$ and $T_{\text {ref }}=273.15 \mathrm{~K}$ (see 
Table 1. Porosities, True Densities, and Bulk Densities for Simulant Materials

\begin{tabular}{lccc}
\hline \multicolumn{1}{c}{ Simulant } & $\phi(\%)$ & $\rho_{\text {bulk }}\left(\mathrm{g} \mathrm{cm}^{-3}\right)$ & $\rho_{\text {true }}\left(\mathrm{g} \mathrm{cm}^{-3}\right)$ \\
\hline 50-80 $\mu$ m glass beads & $44 \pm 1.0$ & $1.46 \pm 0.5$ & 2.50 \\
Crushed JSC Mars-1 & $77 \pm 1.0$ & $1.09 \pm 0.5$ & 3.07 \\
Loose 1-3 $\mu$ m dust & $88 \pm 1.0$ & $0.25 \pm 0.03$ & 2.65 \\
Packed $1-3 \mu \mathrm{m}$ dust & $76 \pm 2.0$ & $0.66 \pm 0.03$ & 2.65 \\
\hline
\end{tabular}

Hudson et al. [2007] for a more complete list). These methods are more accurate than the simple kinetic theory expression, $\mathcal{D}_{12}=\lambda \nu / 3$, where $\lambda$ is the mean free path.

[12] Zalc et al. [2004] compute the Knudsen diffusion coefficient by taking into account deviations from exponential path length distributions and Knudsen cosine law scattering from pore walls, as opposed to random scattering which occurs among gas molecules. Thus, $D_{K}$ may be written as

$$
D_{K}=\frac{1}{3}\left\langle l_{p}\right\rangle\langle v\rangle\left[\frac{\left\langle l_{p}^{2}\right\rangle}{2\left\langle l_{p}\right\rangle^{2}}-\beta\right],
$$

where $\nu$ is the mean molecular velocity $\left(\nu=\sqrt{8 k T / \pi m_{1}}\right)$, $\left\langle l_{p}\right\rangle$ is the first moment of the chord length distribution [Levitz, 1993], and $\beta$ is a series sum of cosine angles between sequential trajectory segments separated by wall collisions. Chords are defined as successive ballistic molecular paths with both ends terminated by a pore wall; therefore, they may be smaller or larger than any individual pore. Note that the Knudsen diffusion coefficient is independent of pressure. From Zalc et al. [2004], the model pore structures used result in nearly exponential chord distributions such that the first term in brackets is near unity. But many real soils may have different distributions, and this quantity can vary significantly. Gille et al. [2001, 2002] describe a method which we employ for determining $\left\langle l_{p}\right\rangle$ from pore diameter distributions determined from mercury porosimetry. The $\beta$ term depends only on the model selected to describe molecular collisions with pore walls [Levitz, 1993]. For the frequently used Knudsen cosine law, this gives $\beta=4 / 13=0.3077$, as confirmed by Zalc et al. [2004] for porosities of $42 \%$ and greater (E. Iglesia, personal communication, 2007), a porosity range which covers all simulants considered in this study except the salt crusts.
[13] The bulk porosity is determined through a gravimetric method detailed in section 3 and summarized in Table 1. Quantitative measurements of the pore size distribution were made for selected simulant materials at a commercial analytical facility; the analysis results are given in section 4.3.

[14] Our setup is not suited for measuring $\mathcal{D}_{12}$ directly, and we therefore use the extrapolation method of Wallace and Sagan [1979], as was done in the work by Hudson et al. [2007] and studies by earlier investigators. However, it is the case that among the different methods, the value of $\mathcal{D}_{12}$ at $250 \mathrm{~K}$ and $600 \mathrm{~Pa}$ varies between 17.5 and $32.0 \mathrm{~cm}^{2} \mathrm{~s}^{-1}$, and so there is some uncertainty in the later tortuosity calculations arising from these differences. The tortuosity analysis results are given in section 5.5.

\section{Experimental Materials}

[15] A variety of materials have been used to explore the effects of particle size distribution and salt crust formation on diffusion coefficients in simulated Mars soils. Properties of these samples and preparation methods are described in this section. Figure 1 shows optical micrographs of the simulants at the same scale. Prior to use, all samples are dried in an oven at $110^{\circ} \mathrm{C}$ and then stored in airtight containers.

[16] Geometric porosities of the separate materials were determined by weighing a known volume of bulk sample to determine a bulk density, $\rho_{\text {bulk }}$, and taking the ratio of this against the known specific gravity of the individual particles $\left(\phi=1-\rho_{\text {bulk }} / \rho_{\text {true }}\right)$. The results are given in Table 1.

\subsection{Glass Beads}

[17] This simulant is composed of beads of soda-lime glass obtained from AGSCO Corporation. They exhibit a narrow particle size distribution between 50 and $80 \mu \mathrm{m}$. The true specific gravity of the glass is 2.50 . To facilitate comparison with our previous experiments, to determine the effect of temperature on advective flux, and to calculate the magnitude of the free gas diffusion correction factor for the temperatures of these experiments, the diffusivity of $50-80 \mu \mathrm{m}$ glass bead samples of $1,2,5$, and $10 \mathrm{~cm}$ thickness were measured. The samples were analyzed by Particle Sizing Systems using a model 780 AccuSizer with lightobscuration techniques and light-scattering techniques.
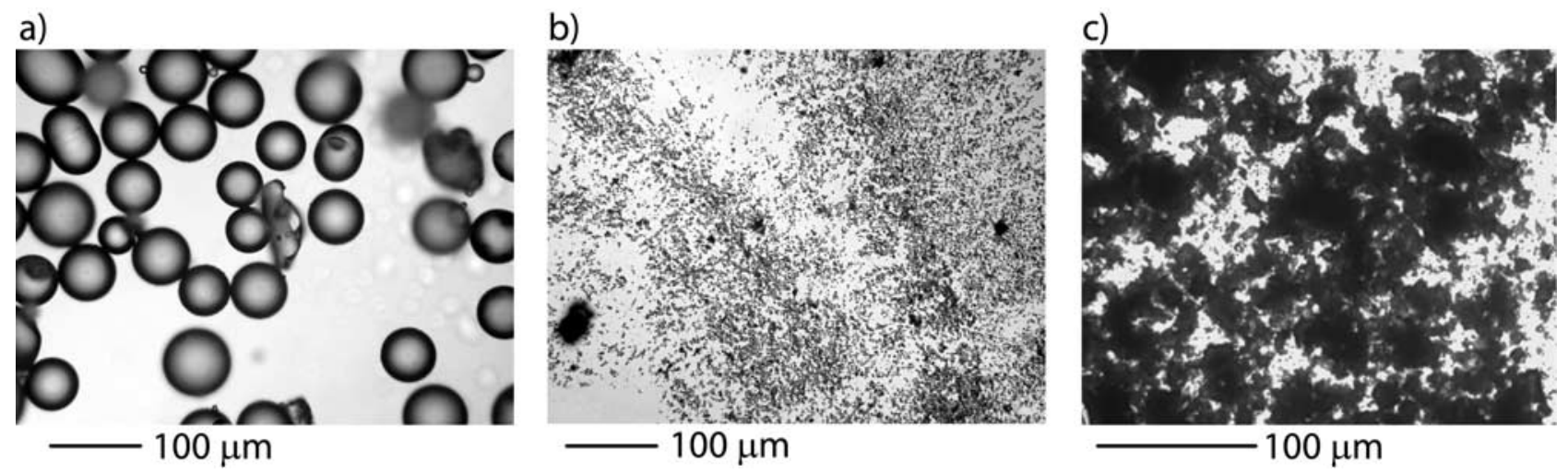

Figure 1. Backlit optical micrographs of regolith simulants of (a) 50-80 $\mu \mathrm{m}$ beads, (b) $1-3 \mu \mathrm{m}$ Arizona Test Dust (note the presence of aggregates), and (c) crushed JSC Mars-1. 



Figure 2. Particle size histogram (solid line) and cumulative finer (dashed-dotted line) plots for (a) crushed JSC Mars-1 and 1-3 $\mu \mathrm{m}$ dust and (b) 50-80 $\mu \mathrm{m}$ glass beads.

Ninety percent of the particles are larger than $48 \mu \mathrm{m}$ and only $10 \%$ are larger than $80 \mu \mathrm{m}$. The volume-weighted mean particle size is $64.4 \mu \mathrm{m}$, with a median of $66.1 \mu \mathrm{m}$ and a mode of $77.7 \mu \mathrm{m}$, reflecting a slight skew toward the larger diameters. The results of the analysis are shown in Figure $2 \mathrm{~b}$. The size of the glass beads places them in the sedimentological category of "fine sand."

\subsection{Crushed JSC Mars-1 Dust}

[18] JSC Mars-1 is a weathered palagonitic cinder-cone ash from Mauna Kea, Hawai'i and has been used in a variety of Mars surface simulation experiments beyond its originally intended use as a spectral analog [Gilmore et al., 2004; Cooper and Mustard, 2002; Gross et al., 2001]. The raw JSC Mars-1 was obtained through the Johnson Space Center Curator. A thorough description of JSC Mars-1 can be found from Allen et al. [1997, 1998].

[19] Raw JSC Mars-1 particles were crushed to smaller sizes in a planetary ball mill. Batches of $250 \mathrm{~mL}$ of ovendried raw material were run with 50 hardened steel milling balls at 590 RPM for $10 \mathrm{~min}$ in the forward direction, rested for $5 \mathrm{~min}$, and milled for another $10 \mathrm{~min}$ in reverse. The resulting particle size distribution was analyzed by Micromeritics Instrument Corporation on a Saturn DigiSizer 5200 using the laser light-scattering technique. Particles were dispersed in a $0.3 \%$ Daxad 23/40\% sucrose/water solution in an ultrasonic bath for $1-2 \mathrm{~min}$. The measured median particle size is $5.46 \mu \mathrm{m}$. The whole distribution is bimodal with peaks at 1.5 and $25.2 \mu \mathrm{m}$; nearly $70 \%$ of the volume is larger than $2 \mu \mathrm{m}$.

[20] An average specific gravity of 1.91 for raw JSC Mars-1 as given by Allen et al. [1998] was quoted in the work by Hudson et al. [2007]. Recent analysis of the crushed sample with He gas displacement in an AccuPyc 1330 Pycnometer at Micromeritics Instrument Corporation yielded an average particle density of $3.07 \mathrm{~g} \mathrm{~cm}^{-1}$. This value is used in subsequent calculations in this paper.

\section{3. $1-3 \mu \mathrm{m}$ Dust}

[21] A fine dust with a very narrow particle size distribution, called "Arizona Test Dust" was obtained from Powder Technology Inc. The dust consists of a natural silicate material with a specific gravity of $2.65 \mu \mathrm{m}$. The dust is composed of equant yet angular particles which easily form weak millimeter- to centimeter-sized aggregates. The presence of larger sand grains, as well as preexperiment and postexperiment sieving, breaks up these aggregates and inhibits their formation.

[22] Loose, aggregated dust alone has a calculated geometric porosity of $88 \pm 1 \%$, while compressional packing results in a reduced porosity of $76 \pm 2 \%$.

[23] The particle size data for this sample, measured on a Coulter Multisizer, were provided by the manufacturer. The mean particle size is $1.19 \pm 0.49 \mu \mathrm{m}$. The median particle size is $1.132 \mu \mathrm{m}$, with less than $1.5 \%$ of the volume in particles larger than $3 \mu \mathrm{m}$. The volume percent and cumulative finer distributions for this simulant and crushed JSC Mars-1 are shown in Figure 2a.

\subsection{Mixtures}

[24] Throughout the rest of this paper, "mixture type 1" will refer to mixtures of crushed JSC Mars-1 material and 50-80 $\mu \mathrm{m}$ glass beads. "Mixture type 2 " will refer to mixtures of $1-3 \mu \mathrm{m}$ silica dust and glass beads.

[25] To study the effect of bimodal particle distributions, particularly the effect of included dust in a deposit of sandsized particles, six mixtures (A through F) of silica glass beads (nominal diameter $50-80 \mu \mathrm{m}$ ) and either crushed JSC Mars-1 or 1-3 $\mu \mathrm{m}$ dust were prepared. The mixtures, both in terms of mass fraction of fines $\left(X_{f}=M_{f} /\left(M_{f}+M_{c}\right)\right)$ and mass ratio of fines to coarse material $\left(M_{f} / M_{c}\right)$ are shown in Table 2. Volume fraction of dust is an indeterminate quantity since $\phi$ and $\rho_{\text {bulk }}$ for the fine materials can vary over a large range, depending on the degree of compaction in the mixture; it is therefore not used to describe the mixtures.

[26] The porosity of the mixtures may be estimated by first calculating the fraction of the volume occupied by each component. Given a measurement of total mixture volume, $V_{T}$, and mixture mass, $M_{T}$, the volume fraction of each is $v_{f}=\left(M_{f} / V_{T}\right)\left(1 / \rho_{f \text {,true }}\right)$ and $v_{c}=\left(M_{c} / V_{T}\right)\left(1 / \rho_{c \text {,true }}\right)$, where $M_{f}$ and $M_{c}$ are the masses of the fine and coarse components as calculated from $M_{f}=X_{f} M_{T}$ and $M_{c}=\left(1-X_{f}\right) M_{T}$. The 
Table 2. Porosities of Mixtures With Glass Beads

\begin{tabular}{lcccccc}
\hline & \multicolumn{6}{c}{ Mixtures of Silica Glass Beads } \\
\cline { 2 - 7 } & $\mathrm{A}$ & $\mathrm{B}$ & $\mathrm{C}$ & $\mathrm{D}$ & $\mathrm{E}$ & $\mathrm{F}$ \\
\hline Mixture type 1 & & & & & & \\
$X_{f}(\%)$ & 43.1 & 27.4 & 15.9 & 11.4 & 7.06 & 3.29 \\
$M_{f} / M_{c}(\%)$ & 75.6 & 37.8 & 18.9 & 12.9 & 7.56 & 3.40 \\
$\rho_{\text {mix }}\left(\mathrm{g} \mathrm{cm}^{-3}\right)$ & 2.72 & 2.63 & 2.58 & 2.55 & 2.53 & 2.52 \\
Mixture type 2 & & & & & & \\
$X_{f}(\%)$ & 39.8 & 24.9 & 14.2 & 10.1 & 6.19 & 2.82 \\
$M_{f} / M_{c}(\%)$ & 66.0 & 33.1 & 16.6 & 11.2 & 6.60 & 2.90 \\
$\rho_{\text {mix }}\left(\mathrm{g} \mathrm{cm}^{-3}\right)$ & 2.56 & 2.54 & 2.52 & 2.51 & 2.51 & 2.50 \\
\hline
\end{tabular}

free volume is then the sum of these volume fractions subtracted from unity:

$$
\phi_{\text {mix }}=1-\frac{M_{T}}{V_{T}}\left[\frac{X_{f}}{\rho_{f, \text { true }}}+\frac{\left(1-X_{f}\right)}{\rho_{c, \text { true }}}\right] .
$$

We determine $\rho_{\text {mix }}$ as proxy for the true density of the mixture if it were composed of a homogeneous granular component: $\rho_{\text {mix }}^{-1}=X_{f} / \rho_{f \text { true }}+\left(1-X_{f}\right) / \rho_{c \text {,true }}$. These values are given in Table 2. Individual porosities are determined for each simulant and are tabulated in the auxiliary material ${ }^{1}$.

[27] The amount of dust included in a mixture may, in addition to its mass fraction of the total, be thought of in terms of the proportion of pore spaces in the dust-free coarse samples which are filled. An attempt to produce a range of mixtures from dust free up to the "critical mixture," where all pore spaces are filled, guided our initial choices of dust mass fractions. The critical mixture concept is, in fact, a less useful definition in practice since the coarse component, in all but the most dust-free mixtures, will be pushed apart by intervening dust particles. Additionally, the dust within the pores may undergo some degree of compaction due to the weight of the overlying material. This effect will be enhanced if coarser (and therefore denser) material is included, as in a mixture. Thus, the value for the bulk density of the fines (needed to calculate the critical mixture) may cover a significant range, even within a single sample.

[28] We expect to see a smooth variation of the measured diffusion coefficient as the mass fraction of dust is increased from pure glass beads to pure dust. Diffusion through pure, unpacked dust in our experiments can be several $\mathrm{cm}^{2} \mathrm{~s}^{-1}$ higher than the value found for glass beads, while for mechanically compacted dust it can be much lower. As stated above, the weight of the dense glass beads should act, at small dust fractions, to compress the dust toward a packed configuration. We do not apply additional mechanical compaction to these samples.

\subsection{Salt Crusts}

[29] Magnesium sulfate was chosen to study the behavior of salt crusts as barrier-forming phenomena because of its availability, crystallization to a well-known phase (epsomite: $\mathrm{MgSO}_{4} \cdot 7 \mathrm{H}_{2} \mathrm{O}$ ) under ambient conditions, and the known existence of sulfate salts on Mars.

[30] Distilled water is saturated with $250 \mathrm{~g}$ of anhydrous $\mathrm{MgSO}_{4}$ per liter and then mixed with a given mass of $40-$ $80 \mu \mathrm{m}$ glass beads and excess distilled water to produce a slurry of dissolved salt and beads. A mold consisting of a

\footnotetext{
${ }^{1}$ Auxiliary materials are available in the HTML. doi:10.1029/ 2007JE003026.
}

plastic ring and support wires (see Figure 3a) is attached to a plastic base with water-resistant grease. The walls and base of this mold are coated with a thin layer of WD-40 lubricant to facilitate removal of the dried crust. Once poured into the mold, the slurry and mold were agitated to remove any trapped air and produce a flat upper surface. Any excess was scraped off and returned to the slurry batch. Slurries were allowed to evaporatively dry in ambient laboratory conditions for $\sim 6 \mathrm{~h}$. Internal cohesiveness then allowed them to be removed from the flat mold base and placed on a coarse wire mesh in a forced draft, thereby continuing to dry from both surfaces of the crust. After $\sim 24 \mathrm{~h}$, when the crusts were completely dry, they were placed in a desiccator and moved into the freezer to thermally equilibrate with the environment prior to the start of an experiment.

[31] Slurries were prepared with $0.5,1.0,2.5,5.0,7.5$, and $10.0 \mathrm{wt} \% \mathrm{MgSO}_{4}\left(\mathrm{MW}=120.37 \mathrm{~g} \mathrm{~mol}^{-1}\right)$. When allowed to crystallize at ambient conditions, epsomite is produced $\left(\mathrm{MW}=246.37 \mathrm{~g} \mathrm{~mol}^{-1}\right)$ giving salt contents of $1.0,2.0,4.9,9.7,14.2$, and 18.4 wt $\%$.

[32] X-ray powder diffraction was performed on the sample crusts before and after exposure to experimental conditions. Most of the X-ray spectrum indicated the presence of an amorphous phase, i.e., the glass beads. The peaks that did occur in the spectrum matched the intensity and location expected for epsomite. Samples of crust which incorporated greater proportions of interior material exhibited weaker peaks, indicating that a higher proportion of salt was present at the outer layers of the crusts. To examine the surface crusts more closely, the top and bottom surfaces of the crust were scraped following an experiment. The bottom surface of the crust (i.e., the surface adjacent to the ice during the experiment) showed the same strong set of epsomite peaks as before exposure to experimental conditions. The upper surface scrapings (i.e., from the surface exposed to the dry chamber atmosphere) exhibited a complete change of spectrum and revealed only the presence of hexahydrite, indicating a loss of the loosely bound seventh water molecule under Mars-like conditions. It is unknown how deeply into the sample this conversion penetrates.

[33] For a $1 \mathrm{~cm}$ thick crust of an initial composition of $14.2 \mathrm{wt} \%$ epsomite, the total amount of epsomite present is $\sim 8.2 \mathrm{~g}$. If it is assumed that one half of the crust converted completely to hexahydrite, the mass of water lost would be $0.3 \mathrm{~g}$. This is less than $4 \%$ of the total amount of mass lost through ice sublimation and contributes a negligible error to the measured flux.

[34] The reconstructive transformation which takes place between epsomite and hexahydrite results in the destruction of large crystals in favor of much smaller crystallites. This microfracturing could increase the available vapor transport volume. We do not constrain the time scale of this transformation under these experimental conditions, but we do observe a linear mass loss rate after initial transients, suggesting a diffusion coefficient which is largely constant in time. This implies two possibilities: Transformation may be rapid, occurring in the initial few hours of exposure to vacuum, and thus the diffusion coefficients we measure are those of glass beads encrusted with fractured, dehydrated salts. The other possibility is that the transformation is gradual, occurring throughout the experimental duration, 

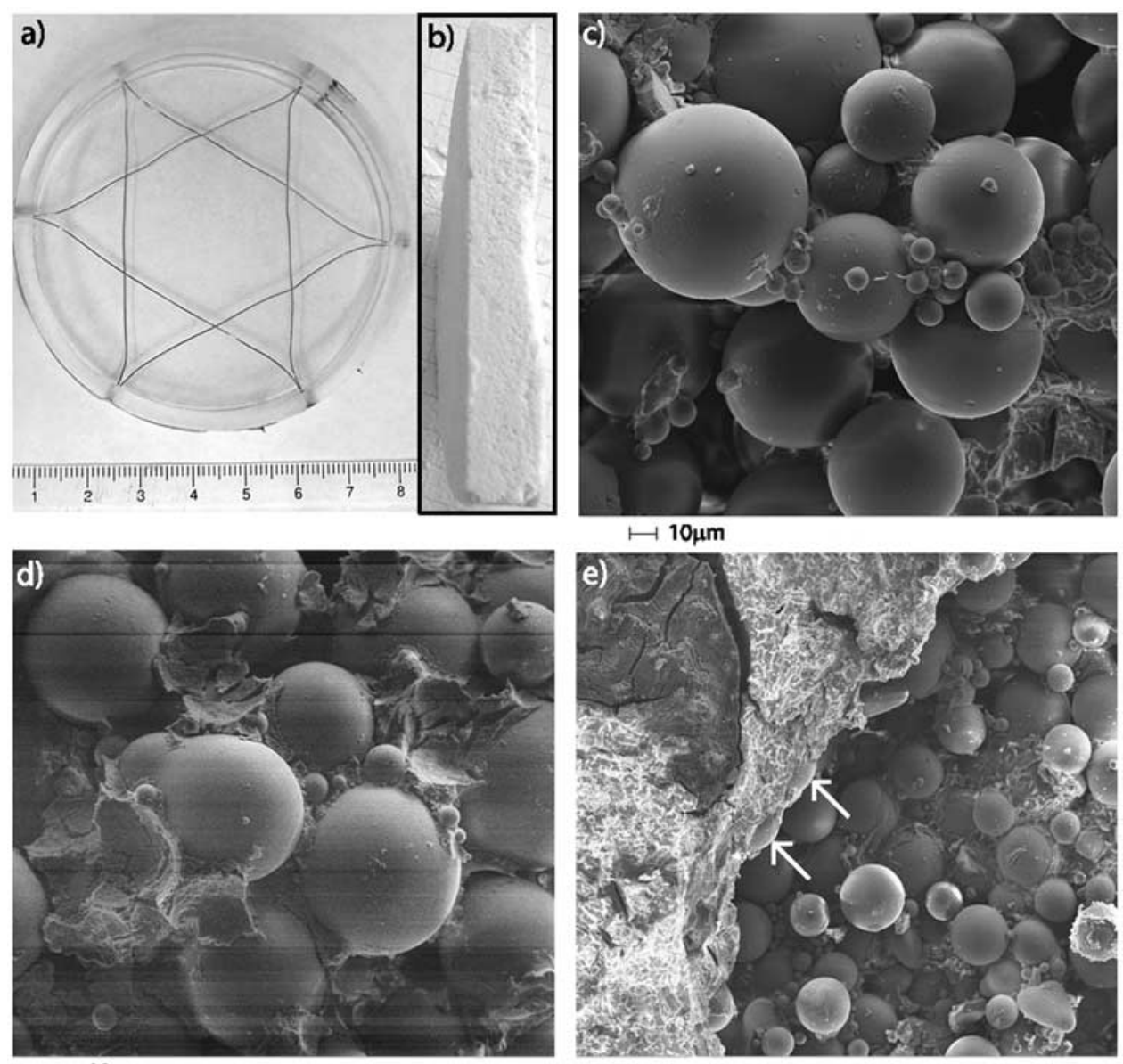

$\longmapsto 10 \mu \mathrm{m}$

$$
\mapsto 20 \mu \mathrm{m}
$$

Figure 3. (a) Empty salt crust caddy (1 cm depth) showing support wires. (b) Cross-section of a crust grown without wires. Figures $3 \mathrm{c}-3 \mathrm{e}$ show scanning electron microscopy images of a $14.2 \mathrm{wt} \%$ epsomite salt crust after exposure to experimental conditions. (c) Interior of crust: view of a cluster of beads held together by salt, beads much smaller than $50-80 \mu \mathrm{m}$ are present in a significant number and frequently fill pore spaces between larger beads. (d) Interior of crust: section of crust showing salt features filling void spaces between larger grains. Dehydration to hexahydrite is the presumed cause of the fracturing. (e) Surface of crust: the left side of the image is dominated by an efflorescent crust of hexahydrite which embeds some beads (arrows) but is predominantly pure salt.

but has an effect on the diffusivity which is small compared to that arising from the overall salt content.

\section{Experimental Description}

[35] Experiments were conducted at the Mars Simulation and Ice Laboratory at the California Institute of Technology. A custom-built stainless steel vacuum chamber contained within a walk-in freezer with a nominal temperature of $250 \mathrm{~K}$ was used to achieve Mars-like pressures and a controlled humidity environment. The experiments were performed with a protocol similar to that described by Hudson et al. [2007]. An abbreviated description highlighting the differences follows.

\subsection{Chamber Setup}

[36] The environmental chamber and instrumental setup are identical to that described by Hudson et al. [2007] in all but the following two respects: (1) The freezer temperature was reduced to give ambient chamber temperatures of $\sim 250 \mathrm{~K}$ rather than $\sim 263 \mathrm{~K}$. (2) Pressure is now maintained more accurately with active proportional integral differential (PID) control of an STEC-4400 mass flow controller via an USB-1408FS data logger from Measurement Computing Corporation.

[37] Important points of the experimental setup are as follows. The chamber's $\mathrm{CO}_{2}$ atmosphere is constantly exchanged and maintained dry at a constant pressure of $586 \mathrm{~Pa}$. Atmospheric vapor content and temperature immediately above the sample are monitored with a combined relative humidity/resistance temperature device (RH/RTD) sensor. Ice temperature is monitored with an embedded Ttype thermocouple. Sample mass is continuously monitored with a wheatstone bridge load cell. Data from two simultaneous experiments are recorded with a Campbell Scientific CR1000 data logger.

\subsection{Sample Preparation and Experimental Method}

[38] All preparation steps following the initial creation of mixtures or salt crusts are performed in a walk-in freezer at 
$263 \mathrm{~K}$. Caddies are weighed before and after sample emplacement so that total mass of simulant, total mass of ice, and total true thickness may be calculated.

[39] The unconsolidated mixtures were placed in $6 \mathrm{~cm}$ tall plastic caddies with $1 \mathrm{~cm}$ of bubble-free ice at the bottom. The media were poured into the caddies from their holding containers until mounding over the rim. The samples were then planed off with a straight edge to match the height of the caddy. Thus, the samples are not actively compressed but may be subject to self-compression. In most cases, the sample surfaces remain within $1 \mathrm{~mm}$ of the caddy top. Some samples with very high dust fractions exhibit some settling as the container is moved from the preparation station to the vacuum chamber. This amounts to about $12 \%$ of the $5 \mathrm{~cm}$ sample depth, at most. The reduced thickness is measured and included in subsequent calculations; additional material is not added.

[40] The $1 \mathrm{~cm}$ salt crusts are placed over an ice-filled, $1 \mathrm{~cm}$ caddy and secured in place with water-resistant grease. Following an experiment with salt crusts, the ice surface is examined to determine if there were any leaks between the ice and salt caddies. (The low-pressure, low-temperature environment gives rise to distinctive ice surface morphologies if the vapor escapes in a rapid or asymmetric way.)

[41] Samples are placed in the chamber, which is sealed and pumped down to $586 \mathrm{~Pa}$ at an initial rate of $\sim 500 \mathrm{~Pa} \mathrm{~s}^{-1}$. Transients due to adsorption of water in the sample or temperature disequilibrium decay after $\sim 2 \mathrm{~h}$. Samples are allowed to evolve for a minimum of $24 \mathrm{~h}$. Longer times are needed than in previous experiments at higher temperatures so that the signal-to-noise ratio of the mass loss curve is high despite the reduced mass loss rates.

[42] Following an experiment, mixture samples are dried at $110^{\circ} \mathrm{C}$ for $24 \mathrm{~h}$, sieved to break up any aggregates which may have formed in the drying process, and recycled to the mixture batch. Salt crust samples are dissolved with distilled water and reused.

[43] Data for calculating diffusion coefficients are taken from the stable interval following the decay of initial transients. The mass loss rate is derived from a least squares linear fit to the measured mass versus time curve. The mass loss rate is then converted to total $\mathrm{H}_{2} \mathrm{O}$ flux. Ice temperature is converted to a vapor pressure of $\mathrm{H}_{2} \mathrm{O}$ at the ice surface by assuming that the layer immediately adjacent to the ice is saturated. Relative humidity is reported by the hygrometer and is converted into a partial pressure of water above the sample using the method detailed by Hudson et al. [2007].

\subsection{Data Analysis}

[44] Unconsolidated samples rest directly on the ice, while crusts have a discernible gap between the ice surface and the bottom of the sample. The vapor-density difference across the sample is derived from measurements made at the ice surface and the position of the hygrometer. These may be different from the true lower and upper boundaries of the porous medium. By assuming that all fluxes are proportional to the density gradient across any given layer (whether free gas or porous medium) and applying the conservation of mass requirement that all fluxes be equal in the absence of ice deposition or loss, we establish a correction term which accounts for diffusion through the simulant-free portions of the column. The derivation of this correction is detailed by Hudson et al. [2007].
[45] The introduced correction term $z_{\text {corr }}$ has units of length. Several measurements of identical samples with different thicknesses are used to determine $z_{\text {corr }}$. The same correction term should apply to all experiments with the same temperature, pressure, and sample type, and may be used to solve for corrected individual values of $D$. This was done for $50-80 \mu \mathrm{m}$ glass beads in the $250 \mathrm{~K}$ environment. The correction term was found to be at most $0.06 \mathrm{~cm}$, or a $6 \%$ correction for a $1 \mathrm{~cm}$ thick sample. The shift in calculated diffusion coefficient is much less than the experimental error, even for the thinnest samples. For this reason uncorrected measurements for salt crusts, though conducted on samples $\sim 1 \mathrm{~cm}$ thick, are accurate within the systematic errors. Mixtures were tested with sample columns of $5 \mathrm{~cm}$, a thickness which has a minimal correction contribution even at the warmer temperatures described by Hudson et al. [2007].

\subsection{Pore Structure}

[46] The porosity of the glass beads was determined with the basic method given in section 3 , while for mixtures it was estimated using equation (6). However, this latter method ignores the dust which may not only fill the interstices of the host sand but also may settle between the sand grains during agitation, thus pushing them apart and increasing the "unfilled" volume. Salt crusts are even less amenable to simple interrogations or estimates of pore volume because of the unknown distribution of epsomite and sand grains following crystallization. Nevertheless, it is possible to determine the obstruction factor, the ratio of porosity to tortuosity, once the diffusion coefficient has been measured.

[47] Four simulants were sent to Porous Materials, Inc. for analysis by mercury porosimetry. This technique reports pore size distributions, median pore diameter, and pore volume and surface area. The simulants chosen for this analysis were pure $50-80 \mu \mathrm{m}$ glass beads, pure crushed JSC Mars-1 dust, and the two most dust-rich varieties of mixture type 1 and mixture type 2 (varieties " $A$ " in Table 2). The pore size distributions for these four materials are plotted in Figure 4.

[48] Figure 4 reveals that the pore size for the $50-80 \mu \mathrm{m}$ glass beads peaks sharply at $\sim 20-25 \mu \mathrm{m}$. All distributions show significant tails at very small pore diameters, i.e., $10 \mathrm{~nm}$ or less. These are likely to represent surface roughness, cracks, and other defects within individual grains in addition to very small passages between grains. While these passages do contribute marginally to the diffusible volume if interconnected, and to the tortuosity if blind, their contribution to the total flux will be negligible. As shown by Clifford and Hillel [1983], where pore sizes down to $1 \mathrm{~nm}$ are considered, pore distributions containing even a few percent porosity in larger (i.e., micron-sized) pores experience most of their diffusive flux through these large spaces.

[49] Examining the individual distributions reveals some interesting features. In crushed JSC Mars-1, the peak pore size, which is centered approximately on $1 \mu \mathrm{m}$, is much broader than for the more regular glass beads and the pore distribution goes to zero almost nowhere below $100 \mu \mathrm{m}$. In the mixture containing both glass beads and JSC Mars-1 dust, the modal peak falls between the modal peaks of the 


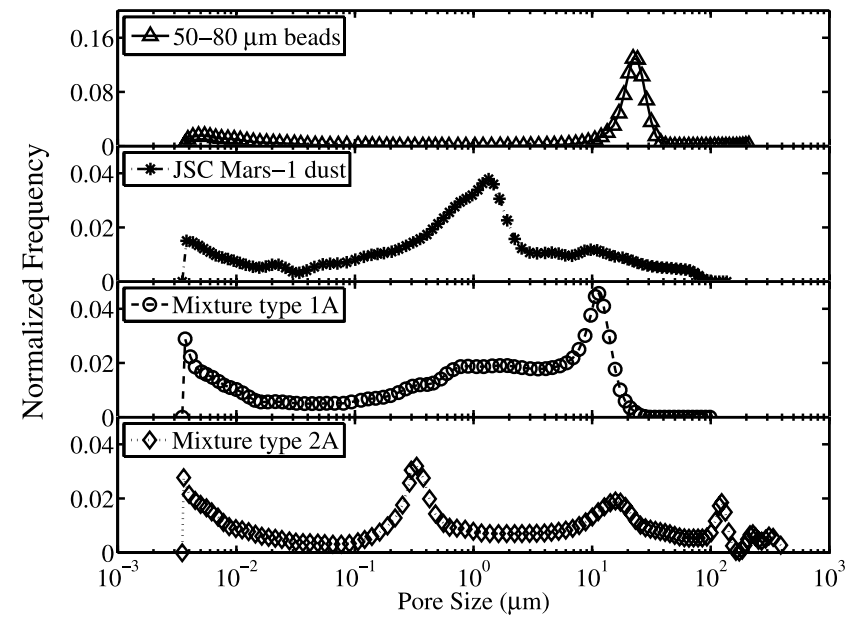

Figure 4. Normalized pore size distribution functions for four samples analyzed with mercury porosimetry.

two mixture components at $\sim 10 \mu \mathrm{m}$. The pore diameter distribution in this material drops sharply to the right of this peak, being zero above $30 \mu \mathrm{m}$. This suggests that selfcompaction and the availability of small grains acts to close off the largest pore spaces. Comparing the mixture of beads and JSC Mars-1 to the mixture composed of beads and $1-3 \mu \mathrm{m}$ dust further underscores the effects of selfcompaction. The micron-sized dust is much less dense than that of the crushed JSC Mars-1 and does not compress as readily under its own weight. The resulting multimodal distribution not only has pore spaces indicative of close packing of dust grains (i.e., the peak at $0.3 \mu \mathrm{m}$ ) but also possesses a very significant fraction of pores greater than $100 \mu \mathrm{m}$ across. In a material containing such large pores, which might be termed a "fairy-castle" structure, these open spaces will accommodate the majority of the diffusive flux. However, these large pore spaces are unlikely to be connected as a high-diffusivity conduit throughout a thick porous medium; transport will be governed by the average resistive path experienced by a diffusing molecule which shall include segments of less open material. It should be cautioned that mercury porosimetry is a highpressure intrusive technique and may result in a rearrangement of pores and particles; very fragile structures may be disrupted.

[50] Of these four simulants, the one with the distribution which appears to have the lowest amount of large crosssection pore space available for transport is the $40 \%$ massfraction fines mixture of glass beads and crushed JSC Mars-1 dust. Effects that may be responsible for closing off large pores include altered bulk density, which enhances self-compaction, and the availability of small particles to fill in the interstices. For further analyses of a broader selection of particle sizes and shapes, the reader is referred to the recent work of Sizemore and Mellon [2007]. In section 5.5 we use our porosimetry measurements to estimate the tortuosity for these four samples.

\section{Results}

[51] This section details the results of our investigations of diffusive properties of glass beads, salt crusts, two types of bead and dust mixtures, and two types of pure dusts under Mars-like conditions. Figure 5 shows the vapor fluxes as a function of temperature along with the evaporation rate curve for bare ice as given by Ingersoll [1970] for a dry atmosphere. Slightly different background temperatures used during the experiments result in the offset indicated by the arrows. The shift is nearly horizontal, indicating that flux is relatively insensitive to these small changes in absolute temperature. Data for individual experiments are provided in the online auxiliary material.

\section{1. $50-80 \mu \mathrm{m}$ Glass Beads at $250 \mathrm{~K}$}

[52] Glass beads are the main component which comprises most of our more complex simulants. Measurement of the compositionally and geometrically simple glass beads facilitates comparison with previous experiments [Hudson et al., 2007]. The beads are all nearly perfect spheres, 50$80 \mu \mathrm{m}$ in diameter. The true particle size distribution is shown in Figure 2b. Within a factor of 2 in friction threshold velocity, these are similar to the $100 \mu \mathrm{m}$ size of the most easily lofted particles under Martian conditions [Greeley et al., 1980].

[53] The dominant size of the beads is $50-80 \mu \mathrm{m}$, but the distribution has a significant tail down to smaller sizes as revealed in Figure $2 \mathrm{~b}$ and the scanning electron microscopy images in Figures $3 \mathrm{c}$ and 3d. For closely packed particles with such a size distribution, the minimum pore size is of the order of $10 \mu \mathrm{m}$ and the maximum is $\sim 80 \mu \mathrm{m}$ [Hudson et al., 2007]. With a mean free path of $\sim 11.5 \mu \mathrm{m}$ under these experimental conditions, diffusion should be dominated by Fickian processes of molecule-molecule collisions, but Knudsen interactions will contribute to an observable extent. The data for glass beads at $250 \mathrm{~K}$ in the work by Hudson et al. [2007] are included in the data presented here. The weighted mean of the corrected diffusion coefficients is $3.69 \mathrm{~cm}^{2} \mathrm{~s}^{-1}$, with a weighted standard deviation of $0.24 \mathrm{~cm}^{2} \mathrm{~s}^{-1}$. As mentioned in section 4.3, the correction term for this simulant is $z_{\text {corr }} \leq 0.6 \mathrm{~mm}$. Figure 6 shows both the raw and corrected values. All raw values greater than $2 \mathrm{~cm}$ thicknesses fall within one standard deviation of the weighted mean.

[54] If we use the Fickian dependence of diffusion coefficient on temperature and pressure of $T^{3 / 2}$ and $P^{-1}$, respectively, and choose a typical Mars temperature of $200 \mathrm{~K}$ and pressure of $600 \mathrm{~Pa}$, we obtain $D(200 \mathrm{~K})=$ $2.73 \pm 0.28 \mathrm{~cm}^{2} \mathrm{~s}^{-1}$. The glass beads measured in these experiments have a mean geometric porosity of $44 \pm 2 \%$, as determined from volume and mass measurements of the bulk sample and knowledge of the true density of the particles.

\subsection{Epsomite Salt Crusts}

[55] The presence of sulfate salts on Mars, the frequency of observed duricrusts, and the potential of both to reduce soil porosity motivate their investigation. As convenient and consistent samples, glass beads cemented with magnesium sulfate salt are created in the laboratory, and the diffusion coefficient is measured for a range of salt contents. Some inhomogeneities are observed in the salt crusts when they are broken following experiments. Outer layers of the crusts tend to be more cohesive than the center in samples with 


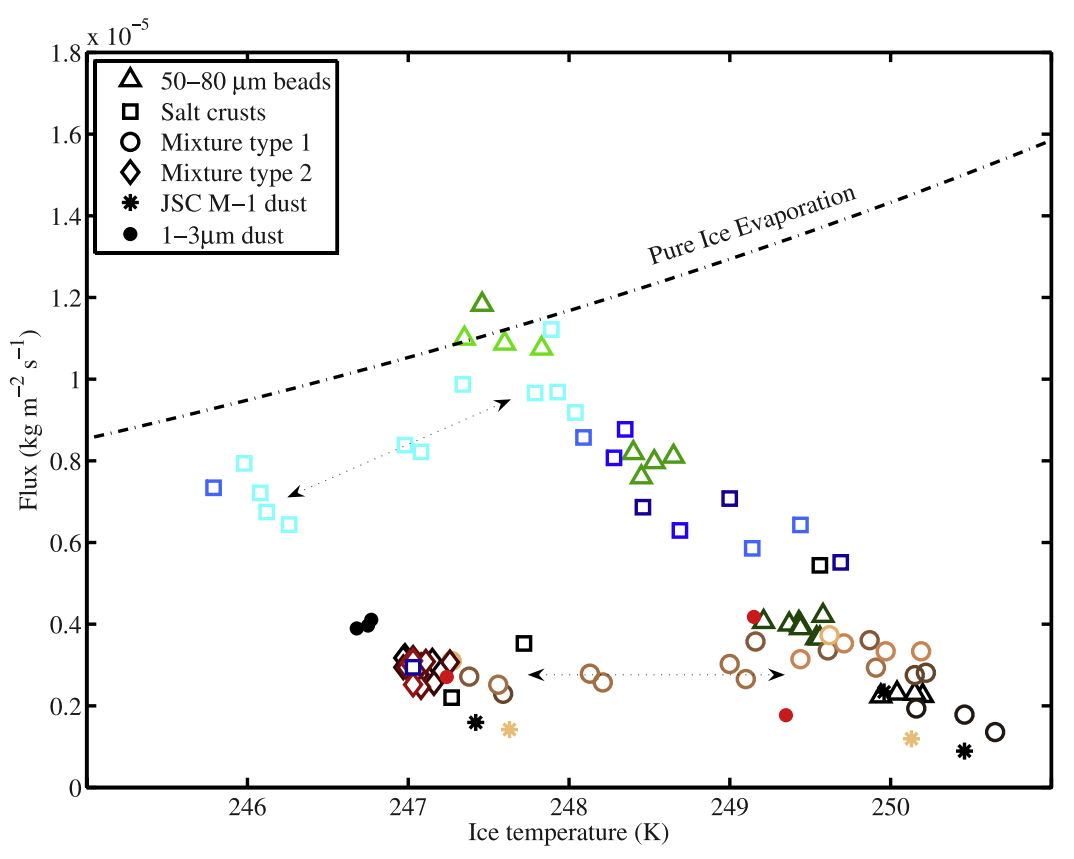

Figure 5. Flux versus ice temperature for each experiment; errors are smaller than the symbols. The dashed-dotted line is the theoretical curve for evaporation of free ice into a dry atmosphere [Ingersoll, 1970]. For a given sample, color trends from dark to light denote decreasing resistance to diffusion (e.g., lower thickness, dust content, or salt content). Arrows indicate trends within a single sample type as a function of temperature. See section 3.4 for mixture type definitions.

high salt contents. These layers may account for the majority of the diffusive resistance of the samples.

[56] The diffusion coefficient data for the salt crusts are presented in Figure 7, which shows that the diffusion coefficient for crusts decreases with increasing salt content. The negative trend is expected, and a reduction in the diffusion coefficient by nearly a factor of 8 over that of pure glass beads occurs for salt concentrations of $18 \mathrm{wt} \%$ epsomite. Lacking measurements of the porosity, we cannot calculate a tortuosity for the salt crusts. Obstruction factors vary as the diffusion coefficient, and overall the factor, is reduced from 0.14 to 0.02 between salt-free and the most salt-laden experiments.

\subsection{Mixtures}

[57] The inclusion of small particles within a matrix of larger clasts can reduce the porosity available for vapor transport and has the potential to significantly change the diffusion coefficient [Farmer, 1976]. The diffusion coefficient for mixtures of glass beads with both crushed JSC Mars- 1 and 1-3 $\mu \mathrm{m}$ dust are plotted in Figure 8. For mixture type 1 (glass beads and crushed JSC Mars-1), the diffusion coefficient and obstruction factor decrease strongly with increasing dust mass fraction and while measured porosity increases. However, for mixture type 2 (1-3 $\mu \mathrm{m}$ dust and glass beads), neither diffusion coefficient nor obstruction factor trend strongly with dust mass fraction, but an obvious positive correlation with porosity remains.

[58] The calculated porosity for both types of mixtures are displayed in Figure 9a. Also included are data for the measured porosity of packed samples of both pure crushed JSC Mars- 1 and pure $1-3 \mu \mathrm{m}$ dust. The trend for both types of mixtures as the mass fraction of dust increases is for the total porosity to increase and approach the values exhibited by pure dust. In the case of bead and $1-3 \mu \mathrm{m}$ dust mixtures, this values seems to be reached at $X_{f} \sim 40 \%$, the trend with beads plus crushed JSC Mars-1, if linear, would reach this point at approximately $X_{f} \sim 60 \%$. The overall increase in porosity with dust content indicates that the included dust tends to coat the larger particles upon mixing and acts to keep them apart, thereby substantially increasing the total volume available. This is in contrast to the case where dust might fill the available pore spaces of a coarse medium without increasing the spacing between larger particles.

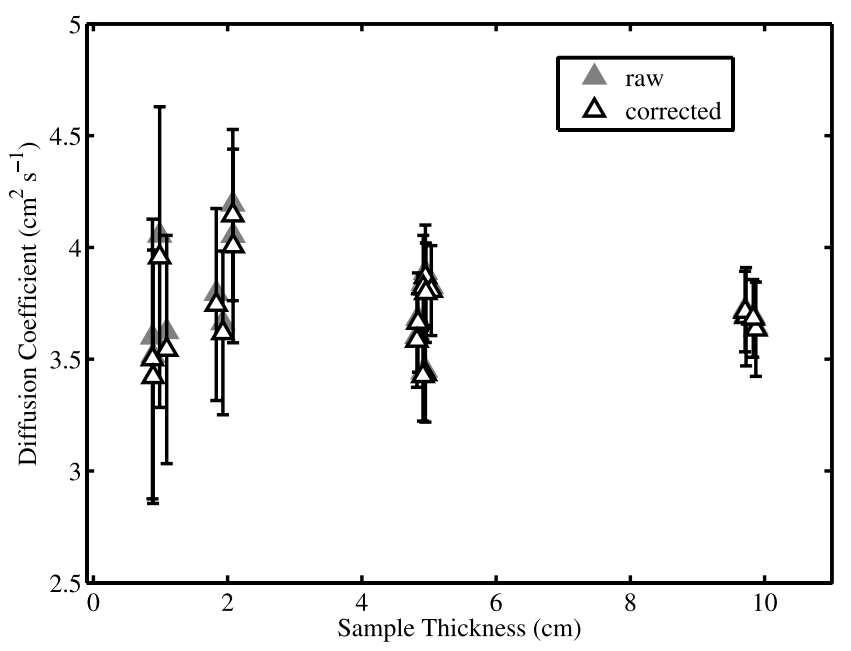

Figure 6. Raw and corrected diffusion coefficients for $50-80 \mu \mathrm{m}$ glass beads at $250 \mathrm{~K}$ and $586 \mathrm{~Pa}$. The correction term is only $0.06 \mathrm{~cm}$; raw values are nearly indistinguishable from corrected values. 


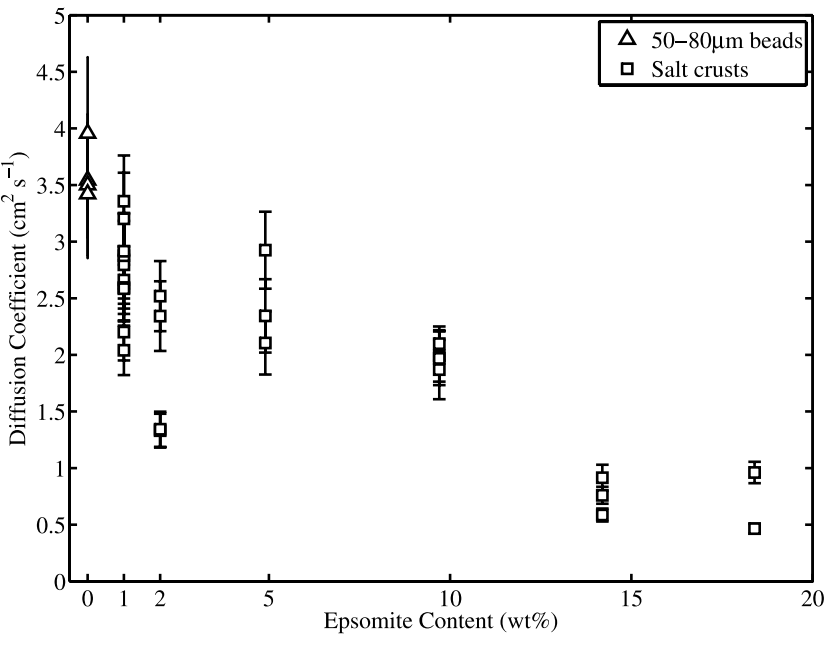

Figure 7. Diffusion coefficients for crusts of 50-80 $\mu \mathrm{m}$ glass beads and $\mathrm{MgSO}_{4} \cdot 7 \mathrm{H}_{2} \mathrm{O}$ at $250 \mathrm{~K}$ and $586 \mathrm{~Pa}$.

[59] The value of the obstruction factor for all simulants is equal to the diffusion coefficient divided by the free gas diffusivity, a quantity which is constant in the experimental setup. The large increase in measured porosity for the micron-sized dust mixtures is counterbalanced by an apparent minor increase in the value of tortuosity, such that the obstruction factors hardly change with $X_{f}$. For mixtures containing beads and crushed JSC Mars-1, however, the increase in porosity is apparently superseded by a tortuosity which increases faster, leading to a smaller obstruction factor with larger dust mass fraction. Since tortuosity should be constant with diffusion regime, all else being equal, it must be the case that high proportions of micron-scale dust

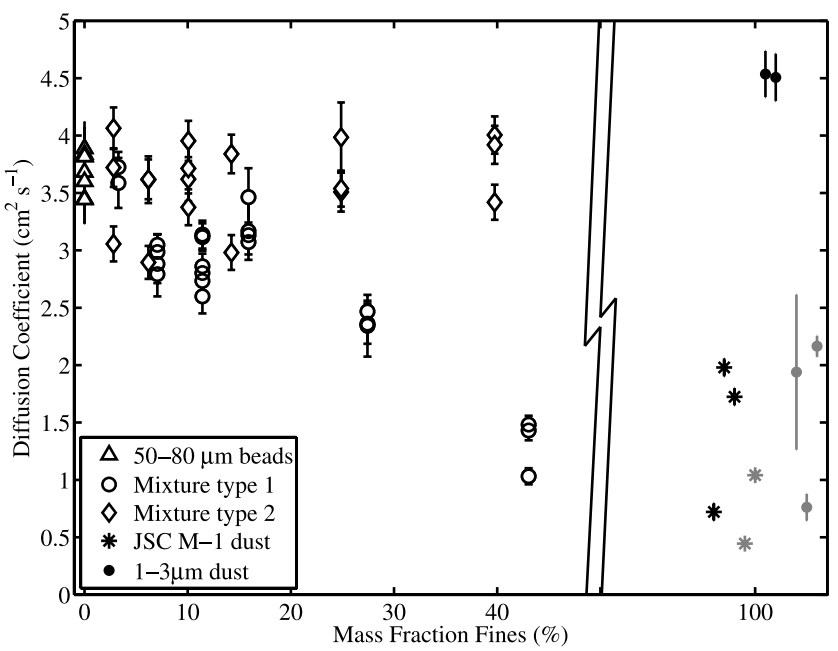

Figure 8. Diffusion coefficients for mixtures of 50-80 $\mu \mathrm{m}$ glass beads with crushed JSC Mars-1 (mixture type 1 (circles)), mixtures of glass beads with $1-3 \mu \mathrm{m}$ dust (mixture type 2 (diamonds)), and diffusion coefficients for pure dusts (asterisks and filled circles). All data are obtained at $250 \mathrm{~K}$ and $586 \mathrm{~Pa}$. Mechanically compacted dust experiments are indicated in gray. All data to the right of the break are $100 \%$ dust by mass, but are separated for clarity.

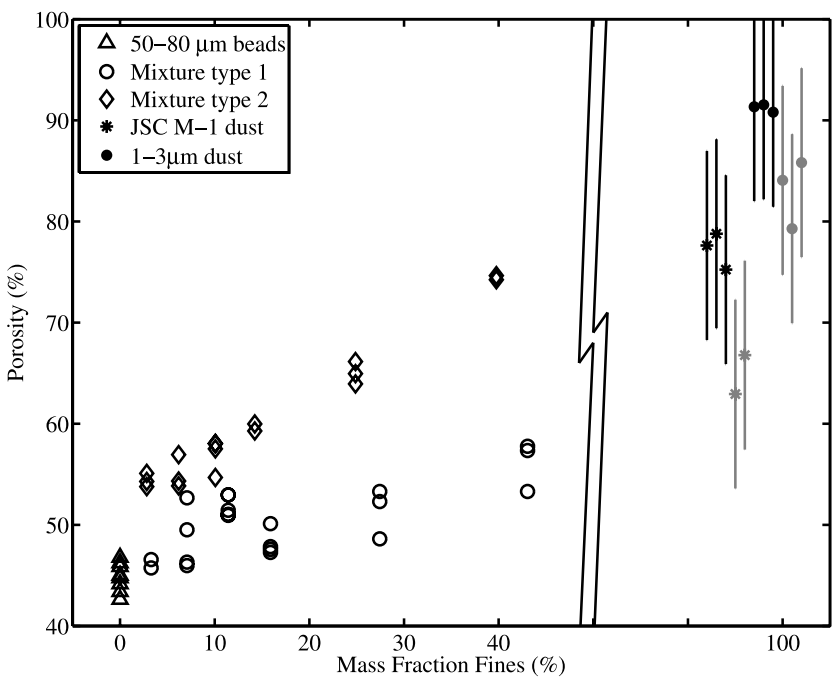

Figure 9. Porosities for pure glass beads (triangles), mixtures of beads and fine dusts (open circles and diamonds), and pure fine dusts (asterisks and closed circles). Mechanically packed dust samples are in gray. All data to the right of the break are $100 \%$ dust by mass, but are separated for clarity. Porosity of the mixtures of glass beads and crushed JSC Mars-1(mixture type 1) increase slowly, while the less self-compacting mixtures containing beads and micron-sized dust achieve higher porosities.

give rise to small pores, changing the overall structure and producing a greater tortuosity.

\subsection{Dusts}

[60] We examined pure samples of the $1-3 \mu \mathrm{m}$ dust and crushed JSC Mars-1 in both packed and unpacked configurations. Loose dust may be considered a crude approximation to airfall dust, while compaction simulates the effect of burial.

[61] Loose dust was poured into the sample caddy and planed off to $5 \mathrm{~cm}$ thickness. The low-density, $1-3 \mu \mathrm{m}$ dust exhibited no observable self-compaction, even in samples of this thickness. Both dust types were seen to deposit with visible submillimeter gaps between aggregates. Though aggregates of the size and density observed in this sample handling method are unlikely to be supportable by the thin Martian atmosphere, airfall dust deposits on Mars will likely form low-density aggregates in response to electrostatic forces, resulting in deposits of even lower bulk density.

[62] For compacted samples, the equivalent of between 5 and $8 \mathrm{~cm}$ of loose dust was pressed into the sample caddies with a flat, circular plate. Air in the pore spaces was allowed to escape around the edges of the plate. Following compaction, the sample was weighed to determine a bulk density and total thickness.

[63] The experiments show that packed and unpacked JSC Mars-1 has a range in diffusion coefficient from $0.45-$ $1.98 \mathrm{~cm}^{2} \mathrm{~s}^{-1}$, with a weighted mean value of $0.77 \pm$ $0.04 \mathrm{~cm}^{2} \mathrm{~s}^{-1}$. Both sets of values are included in these numbers since their ranges overlap. The $1-3 \mu \mathrm{m}$ dust exhibits a larger distinction between loose and compacted samples, with their weighted mean values being $4.71 \pm 0.22$ and $1.64 \pm 0.11 \mathrm{~cm}^{2} \mathrm{~s}^{-1}$, respectively. 


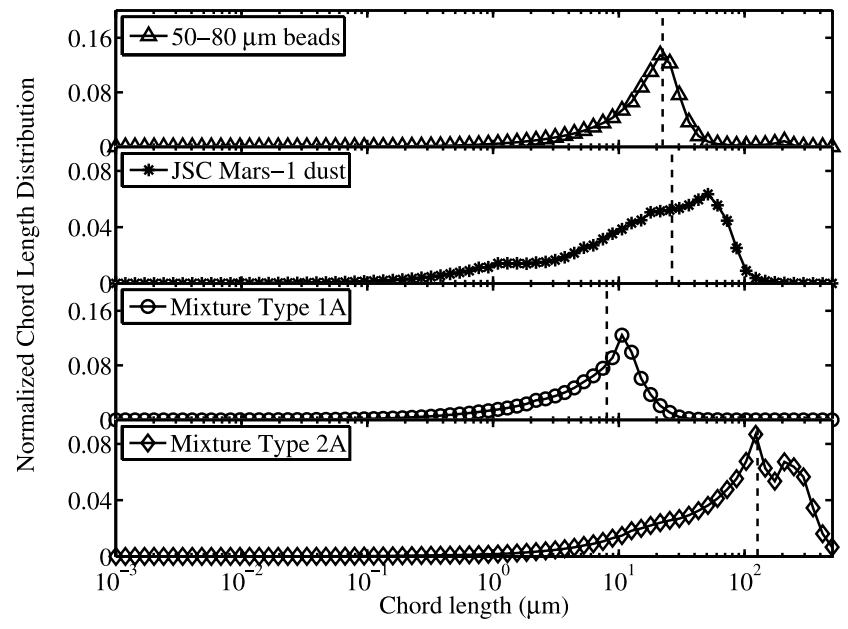

Figure 10. Normalized chord length distributions functions computed from pore size distributions. Vertical dashed lines are first moments of the chord length distributions, $\left\langle l_{p}\right\rangle$.

\subsection{Sample Tortuosity}

[64] Hudson et al. [2007] determined independent Fickian and Knudsen diffusion coefficients and calculated a tortuosity for $50-80 \mu \mathrm{m}$ glass beads of $1.8 \pm 0.6$. This compares favorably with earlier measurements of tortuosity of glass spheres such as those by Hoogschagen [1955], who determined $\tau=1.3-1.5$ and Currie [1960], who theoretically calculated values between 1.3 and 1.7 and experimentally found $\tau=1.4-2$ for large spheres $(380 \mu \mathrm{m}$ to $6 \mathrm{~mm})$.

[65] Here we use the method of Zalc et al. [2004] described in section 2 to estimate $D_{K}$ and then the geometric (i.e., independent of diffusion regime) tortuosity for the four selected samples of section 4.3. This calculation takes as inputs the measured effective diffusion coefficient, porosity, free gas diffusion coefficient, and the chord length distribution function, $p(l)$.

[66] To retain consistency with our earlier work and with the work of a number of previous investigators, we use the method of Wallace and Sagan [1979] to determine the free gas diffusion coefficient, $\mathcal{D}_{12}$, noting that other calculation methods produce values that are different from this by as much as a factor of 2 but are typically closer.

[67] The chord length distribution is calculated from the pore size distribution, $V(x)$, using the method described by Gille et al. [2002], which approximates the pore spaces as cylinders of varying lengths. Thus, $p(l)$ is given by

$$
p(l)=\frac{\int_{0}^{L} x \cdot A_{0}(l, x) \cdot V(x) \mathrm{d} x}{\int_{0}^{L} x \cdot V(x) \mathrm{d} x},
$$

where $l$ is random chord length within a pore, $x$ is the pore diameter, $L$ is a length parameter, and $A_{0}(l, x)$ is the chord length distribution of an infinitely long cylinder of diameter, $x . A_{0}(l, x)$ is given by Gille et al. [2001] as expressions involving Gauss' hypergeometric functions, ${ }_{2} F_{1}$ :

$$
\begin{gathered}
A(l, x)=\frac{3 l}{4 x^{2}} \cdot 2 F_{1}\left(\frac{1}{2} ; \frac{5}{2} ; 3 ; \frac{l^{2}}{x^{2}}\right), l<x \\
=\frac{3 x^{3}}{4 l^{4}} \cdot 2 F_{1}\left(\frac{1}{2} ; \frac{5}{2} ; 3 ; \frac{x^{2}}{l^{2}}\right), l>x .
\end{gathered}
$$

The assumption of cylindrical pores may be violated in media with irregularly shaped grains.

[68] The first and second moments, $\left\langle l_{p}\right\rangle$ and $\left\langle l_{p}^{2}\right\rangle$, are computed in the standard way. Applying these techniques, we obtain chord length distributions and first moments displayed in Figure 10. Table 3 displays the mean diameter, $\bar{d}$, and first moment of the chord distribution, $\left\langle l_{p}\right\rangle$. Also computed and tabulated is the quotient $\left\langle l_{p}^{2}\right\rangle / 2\left\langle l_{p}\right\rangle^{2}$, which describes the deviation of the chord length distribution function from an exponential distribution, and the calculated tortuosity, $\tau$.

[69] As Table 3 illustrates, the value of $\tau$ thus determined for glass beads is close to the expected values of $\sim 1.5$. The other tortuosity values cover a range up to 6 , which is high compared to ranges used in early estimates of tortuosity (e.g., 3-5 from Smoluchowski [1968]) and to more recent experimental studies [Sizemore and Mellon, 2007]. Uncertainty in these numbers arises both from the variation in $\mathcal{D}_{12}$ and the possible violation of the cylindrical pore assumption. Taken together, these tortuosity estimates are not appreciably different from those used in previous diffusion studies, but their variation underscores the uncertainty of this quantity for many porous materials and highlights the importance of measuring the effective diffusivity directly. It would be useful to further explore the effects of particle size mixtures and salt content on pore structure and pore size distribution and to determine the conditions which give rise to the greatest change in soil diffusive properties.

\subsection{Summary}

[70] Table 4 presents a summary of the data for each type of simulant. For mixtures and salt crusts, the range of observed values of $D, \phi$, and $D / \mathcal{D}_{12}$ are presented. Other simulants show weighted means and standard deviations.

[71] We apply correction terms to experiments performed on glass beads at $250 \mathrm{~K}$ under $\mathrm{CO}_{2}$, though this correction $\left(z_{\text {corr }}=0.6 \mathrm{~mm}\right)$ results in a comparable but smaller shift in the data than systematic scatter and formal errors. Corrections for salt crusts are determined to be unnecessary because the correction term for pure glass beads of the same thickness was found to be negligible at these temperatures. All other experiments are performed with $5 \mathrm{~cm}$ thick

Table 3. Porosities, Average Pore Diameters, First Moments of the Chord Length Distributions $\left(\left\langle l_{p}\right\rangle\right)$, Exponential Distribution Deviation Term, and Tortuosities for Four Samples Analyzed With Mercury Porosimetry

\begin{tabular}{lccccc}
\hline \multicolumn{1}{c}{ Simulant } & $\phi(\%)$ & $\bar{d}(\mu \mathrm{m})$ & $\left\langle l_{p}\right\rangle(\mu \mathrm{m})$ & $\left\langle l_{p}^{2}\right\rangle / 2\left\langle l_{p}\right\rangle^{2}$ & 1.501 \\
\hline $50-80 \mu \mathrm{m}$ beads & 44 & 18.74 & 22.29 & 1.002 & 1.66 \\
Crushed JSC Mars-1 & 77 & 5.35 & 26.46 & 0.794 & 1.01 \\
Mixture type 1A & 56 & 4.42 & 126.65 & 0.903 & 3.82 \\
Mixture type 2A & 75 & 32.7 & & \\
\hline
\end{tabular}


Table 4. Summary of Results for Experiments Carried Out at $250 \mathrm{~K}$ and $\sim 586 \mathrm{~Pa}$ in $\mathrm{CO}_{2}{ }^{\mathrm{a}}$

\begin{tabular}{lccccrr}
\hline \multicolumn{1}{c}{ Sample } & $T_{\text {ice }}(\mathrm{K})$ & $D\left(\mathrm{~cm}^{2} \mathrm{~s}^{-1}\right)$ & $D^{\mathrm{b}}\left(\mathrm{cm}^{2} \mathrm{~s}^{-1}\right)$ & $\phi(\%)$ & $\mathcal{D}_{12}\left(\mathrm{~cm}^{2} \mathrm{~s}^{-1}\right)$ & $D / \mathcal{D}_{12}$ \\
\hline $50-80 \mu \mathrm{m}$ beads & $249.0 \pm 0.4$ & $3.69 \pm 0.24$ & $2.57 \pm 0.16$ & $44 \pm 2$ & $24.5 \pm 3.0$ & $0.150 \pm 0.014$ \\
Beads $+\mathrm{MgSO}_{4} \cdot 7 \mathrm{H}_{2} \mathrm{O}$ & $247.9 \pm 0.4$ & $0.47-3.36$ & $0.34-2.34$ & N/A & $24.8 \pm 1.5$ & $0.019-0.133$ \\
Beads + JSC Mars-1 & $249.4 \pm 0.4$ & $1.03-3.73$ & $0.74-2.68$ & $46-58$ & $25.0 \pm 1.6$ & $0.041-0.150$ \\
Beads $+1-3 \mu$ m dust & $247.1 \pm 0.2$ & $2.90-4.06$ & $2.11-2.90$ & $54-66$ & $24.8 \pm 1.5$ & $0.117-0.160$ \\
JSC Mars-1, loose & $246.7 \pm 0.2$ & $1.47 \pm 0.08$ & $1.03 \pm 0.06$ & $77 \pm 9$ & $24.7 \pm 1.5$ & $0.060 \pm 0.006$ \\
JSC Mars-1, packed & $247.5 \pm 0.2$ & $0.59 \pm 0.03$ & $0.42 \pm 0.02$ & $65 \pm 9$ & $25.9 \pm 1.6$ & $0.023 \pm 0.002$ \\
$1-3 \mu$ m dust, loose & $248.9 \pm 0.2$ & $4.71 \pm 0.22$ & $3.36 \pm 0.16$ & $83 \pm 9$ & $24.9 \pm 1.5$ & $0.190 \pm 0.015$ \\
$1-3 \mu$ m dust, packed & $248.6 \pm 0.2$ & $1.64 \pm 0.11$ & $1.16 \pm 0.08$ & $91 \pm 9$ & $25.1 \pm 1.5$ & $0.062 \pm 0.006$ \\
\hline
\end{tabular}

${ }^{a}$ Weighted averages and standard deviations are given when samples are approximately uniform. Minimum to maximum intervals are given where variation among samples is responsible for the range of observations.

${ }^{\mathrm{b}}$ Diffusion coefficients extrapolated to $200 \mathrm{~K}$ and $600 \mathrm{~Pa}$ using $D \propto T^{3 / 2} P^{-1}$, as appropriate for Fickian diffusion.

samples, which provide enough diffusive resistance to reduce the correction term below systematic errors; the values reported are unmodified.

[72] Diffusivities for all simulants fall within the range of $0.47-4.7 \mathrm{~cm}^{2} \mathrm{~s}^{-1}$. Extrapolation to the Mars-appropriate conditions of $200 \mathrm{~K}$ and $600 \mathrm{~Pa}$ via the Fickian dependence of the diffusion coefficient on temperature and pressure yields the range $0.34-3.30 \mathrm{~cm}^{2} \mathrm{~s}^{-1}$. The obstruction factor for most simulants is between 0.02 and 0.20 . Tortuosities for four simulants cover a range of 1.5-6.

\section{Discussion}

\subsection{Experimental Errors}

[73] The experimental errors allow determination of variations between experimental runs. Hudson et al. [2007] showed that thermodiffusion is less than concentration diffusion by a factor of $0.8\left(m_{2} / m_{1}\right)(\Delta T / T)\left(p_{1} / \Delta p_{1}\right)$. The largest value of $\Delta T / T$ for any experiment is 0.015 , while the average value is 0.009 . For $\left(p_{1} / \Delta p_{1}\right)$, the extreme and average values are 1.8 and 1.2 . Thus, the maximum expected contribution from thermodiffusion is $5 \%$, while more typical values are closer to $2 \%$. Also, Hudson et al. [2007], gave an expression of $0.6\left(\Delta p_{0} / p_{0}\right)$ for the ratio of barodiffusion to concentration diffusion. The maximum possible $\Delta p_{0}$ is the saturation vapor pressure of water at $250 \mathrm{~K}(76 \mathrm{~Pa})$, thus giving a maximum barodiffusion contribution of $7 \%$. The value will be smaller for samples with higher permeability and, therefore, smaller pressure gradients.

[74] Systematic errors may arise from nonuniformities in the experimental setup. The largest variation in measured quantities among samples of a given thickness is the relative humidity, which may vary by between 20 and 36\%. Repositioning or substituting sample vessels may affect the airflow of dry and moist gases in the overturning chamber atmosphere. Water vapor densities at the surface of the sample may not be uniform in the horizontal or vertical directions, and the relative humidity measured by the hygrometer may not reflect the environment across the surface. To address these uncertainties, a large number of experiments have been run to allow us to give confident statistical bounds on our measured diffusion coefficients.

\subsection{Advection}

[75] The corrected diffusion coefficient found for the glass beads in the experiments performed at $250 \mathrm{~K}$ is $3.69 \pm$ $0.24 \mathrm{~cm}^{2} \mathrm{~s}^{-1}$. By comparison, the value obtained by Hudson et al. [2007] for these same simulants at $263 \mathrm{~K}$ is $4.49 \pm$
$0.69 \mathrm{~cm}^{2} \mathrm{~s}^{-1}$. Adjusted for the $12 \mathrm{~K}$ temperature difference using a $T^{3 / 2}$ dependence and a $P^{-1}$ correction for the overall $20 \mathrm{~Pa}$ pressure difference, this latter value would be $4.13 \pm$ $0.64 \mathrm{~cm}^{2} \mathrm{~s}^{-1}$ at $250 \mathrm{~K}$. The lower vapor pressures should result in a lower advective contribution in the colder chamber. Further reducing the $263 \mathrm{~K}$ experiment value by $3 \%$ (as calculated in section 2) gives $4.00 \pm 0.62 \mathrm{~cm}^{2} \mathrm{~s}^{-1}$, a value whose center is much closer to that measured in the $250 \mathrm{~K}$ chamber. However, since the error ranges of the two diffusion coefficients overlap, concluding that advection is responsible for the difference is not possible using these data.

\subsection{Salts and Salt Crusts on Mars}

[76] The existence of salts on the surface of Mars is well established [Clark and van Hart, 1981; Vaniman et al., 2004; Yen et al., 2005], as is their association with surface crusts [Landis et al., 2004; Wang et al., 2006]. Chevrier and Mathé [2007, and references therein] use lines of evidence from in situ and remote sensing observations to conclude that high sulfur abundances (compared to the surface of the Earth) are found over most of the known Martian surface and that sulfur, particularly in the form of sulfate, is an important component in the evolution of the Martian regolith.

[77] The mobility of these salts and their observed ability to cement soil grains imply a potential for pore restriction or closure and a concomitant reduction in the diffusivity of the salt-bearing soils and crusts. Recent observations by the Mars Exploration Rovers (MER) indicate the possibility of two populations of salt-bearing soils: lightly cemented surface duricrusts containing a few percent of salt and more loosely consolidated subsurface deposits of light-toned material which may be as much as $50 \mathrm{wt} \%$ sulfate salt [Cabrol et al., 2006; Yen et al., 2007; Wang et al., 2007].

[78] Up to $50 \mathrm{wt} \%$ iron sulfate is inferred at the Paso Robles site observed by Spirit [Cabrol et al., 2006]. Many other sites present indications that magnesium sulfate is the dominant phase. Greater than $20 \mathrm{wt} \%$ in the wall of the Boroughs trench in Gusev crater was determined to be $\mathrm{Mg}$ and $\mathrm{Ca}$ bearing sulfate. The $\mathrm{Mg} / \mathrm{Ca}$ ratio is high; approximately 5 .

[79] Analyses of sulfate stability fields under Mars surface conditions suggest that the most likely hydrated forms of magnesium sulfate are the monohydrate kieserite or amorphous phases with from 1.2 to $2 \mathrm{~mol}$ units of water. Metastability may permit the persistence of more hydrated phases (e.g., starkeyite, $\mathrm{MgSO}_{4} \cdot 4 \mathrm{H}_{2} \mathrm{O}$ ) which may have originally formed under more favorable climate conditions. The existence of sulfate phases common on Earth (6-hydrate 
hexahydrite or 7-hydrate epsomite) in the shallow subsurface of Mars is unlikely at present [Vaniman et al., 2004].

[80] Regardless of their hydration state, salt crystals may form barriers to diffusion in Mars soils just as they do on Earth. Caliche, a calcium-bearing mineral cement, frequently occurs in terrestrial desert environments. Caliche layers are often sufficiently resistive that they completely impede the movement of water, resulting in perched water tables or aquacludes, the disruption of which produces artesian springs. Such phenomena have been hypothesized to occur on Mars as part of gully formation mechanisms [Malin and Edgett, 2000]. Though magnesium-bearing salts are rather water soluble $\left(56.0 \mathrm{~g} / 100 \mathrm{~g}\right.$ water for $\mathrm{MgCl}_{2}, 35.7 \mathrm{~g} / 100 \mathrm{~g}$ for $\mathrm{MgSO}_{4}$ [Lide, 2003]) and would not form effective barriers to liquid water, they could easily impede or prevent the movement of vapor molecules.

[81] In contrast to the sulfate-rich subsurface deposits observed by the rovers in isolated localities, indurated surface soils, called duricrust, have been observed at all Mars landing sites and are believed to be extensive [Presley and Christensen, 1989]. The physical properties of surface crusts at the Viking lander sites have been calculated, and similar properties over much of the surface of Mars are predicted [Moore, 1992]. The cohesive strength of surface soils at Viking sites falls in the range of 1 to $11 \mathrm{kPa}$ (the cohesion of dry clayey silts such as dried mud flats is between 10 and $30 \mathrm{kPa}$ ). Crusts at the edge of trenches carved by the Opportunity rover are illustrated by Weitz et al. [2006], and the cementation of soils at Gusev is discussed by Cabrol et al. [2006] and Landis et al. [2004]. Conclusive observations of the exact mineral composition and physical structure of these crusts do not yet exist, but evidence supports that the cohesion of Viking crusts is related to the presence of $\mathrm{SO}_{3}$ and $\mathrm{Cl}$ compounds [Clark et al., 1977]. Observations by the MER instruments reveal large quantities of sulfates at numerous sites. Taken together, these data strongly suggest that salts, particularly sulfate salts, play a large role in duricrust formation. Our laboratory-grown crusts have at most $10 \mathrm{wt} \% \mathrm{MgSO}_{4}$, a fraction smaller than that of the subsurface salts exemplified by the Paso Robles and Tyrone type soils.

[82] In our experiments, a small amount of sulfate salt $(\sim 1 \%)$ produces a small reduction in the measured diffusion coefficient of $50-80 \mu \mathrm{m}$ beads on the order of $1 \mathrm{~cm}^{2} \mathrm{~s}^{-1}$. Further increases in salt content have little apparent effect until $>10 \mathrm{wt} \%$ epsomite, where reductions of $D$ by a factor of 5 to 10 are observed.

[83] As the water in the saturated slurry of our laboratory crusts evaporates during formation, crystals preferentially form at points of low surface energy where nucleation is facilitated, such as grain contact points (see Figure 3e). These crystals cement the grains of the material together once the water has evaporated, resulting in some reduction in porosity. Increasing the salt content may further reduce the porosity, but the majority of vapor transport pathways remain open. Beyond 5 wt \% epsomite, we see a reduction in experimental scatter, which suggests that the overall change in pore geometry has become uniform among samples. As still more salt is added, the final porosity is substantially decreased, tortuosity rises, and $D$ drops sig- nificantly. We have not observed salt crusts in the laboratory which completely block vapor diffusion.

[84] The protocol used for making laboratory crusts involves significant liquid water. This is not representative of any known present-day surface condition on Mars, yet geomorphic and geologic evidence, for example at Meridiani Planum, indicates that standing or subsurface water may have been a significant part of the geologic history in certain regions. The measurements here pertain to several studies invoking crust formation and solute transport processes that require small amounts of water. Cabrol et al. [2006] and Landis et al. [2004] discuss possible processes for the formation of salt crusts in the soils of Gusev crater. They suggest that humidity precipitated as frost during the cold Martian night and low thermal inertia of surface materials and the presence of melting point-lowering salts will result in a small transient liquid phase immediately following sunrise. Prior to evaporation, this liquid will dissolve salts which are then reprecipitated around the soil grains, resulting in crust formation. Such processes, they suggest, may only occur during particular seasons and possibly only during favorable years. Wang et al. [2006] conclude that the total amount of water activity necessary for the formation of Gusev crusts is likely to be low, as suggested by the limited quantity of evaporite deposition at the surface, the limited leaching of minerals by water, and the concentration of soluble elements. It may, therefore, be the case that the subsurface salt deposits and surface crusts are formed at different times, on different time scales, and with significantly different amounts of aqueous activity. The subsurface salts may be the source for a slow upward migration of chemical species, which ultimately form the weak surface crusts. Taken together, the results of Cabrol et al. [2006], Landis et al. [2004], and Wang et al. [2006] indicate that formation mechanisms for salt crusts on Mars exist. Note, however, that they involve less water than the laboratory methods used here.

[85] Intriguingly, the bright deposits revealed in rover wheel tracks have not yet been seen to outcrop anywhere in undisturbed regolith, leading to the hypothesis that their placement and vertical extent may be controlled by the surface-atmosphere interface through evaporation, set by the thermal wave penetration. The diffusion of oxidizing species from the atmosphere or surface layer of the regolith may also affect sulfate deposition. Wang et al. [2007] report long-term observations of two types of light-toned soils (termed "yellowish" and "whiteish") at the Tyrone site during the Spirit winter campaign. The yellowish soils are observed to change spectral character after at least 175 soils exposure to Martian surface conditions, becoming more like the whiteish, light-toned soils. This indicates that the more deeply buried yellowish soils were not in equilibrium with the current environment and are undergoing some sort of chemical change, which Wang et al. [2007] suggest may be dehydration of $\mathrm{Fe}_{2}\left(\mathrm{SO}_{4}\right)_{3} \cdot 7 \mathrm{H}_{2} \mathrm{O}$.

[86] Our experimental results suggest that surface duricrusts which show weak degrees of cementation and likely have $<10 \%$ of soluble salt content will not present a substantial barrier to vapor transport, perhaps at most a factor of 2 reduction over uncemented soil. Higher salt content could lead to greater reductions in the diffusion coefficient, and soil salt contents of the appropriate con- 
centrations have been observed. However, the deep sulfate salts which have thus far been uncovered by MER have appeared as loose subsurface powders with small particle sizes. If conditions elsewhere on Mars have permitted these subsurface salts to become mobilized and cemented, it is possible that the highest barriers to diffusion may be found beneath, rather than at, the surface.

\subsection{Diffusion in Mars Dusts}

[87] Dust is present everywhere on Mars and is a significant part of the present-day climate cycle. It forms an active surface layer which may be lofted and redeposited by dust storms and may also be incorporated deep into the regolith. We examine two types of dust with differing particle size distributions in both mechanically packed and unpacked configurations.

[88] The diffusivity values for packed and unpacked JSC Mars-1 dust samples overlap and cover a range from 0.4 to $2.0 \mathrm{~cm}^{2} \mathrm{~s}^{-1}$, indicating that mechanical packing does little to alter the properties of this simulant and that there is wide variation between successive simulants. The large particle size range for this dust allows larger grains to interlock and create a supporting matrix which retains large pore spaces, even under externally applied compaction. Still, each sample is unique in its precise geometry, and variations among samples exist. The larger spread in diffusion coefficients at $X_{f}=1$ than at lesser dust fractions (see section 6.5) indicates that the presence of larger particles at moderate dust fractions helps create a similar geometry from sample to sample through self-compaction, while the less massive pure dust samples are subject to greater variation. Mechanical packing has a significant effect on $1-3 \mu \mathrm{m}$ dust, decreasing its diffusivity from $4.71 \pm 0.22$ to $1.64 \pm 0.11 \mathrm{~cm}^{2} \mathrm{~s}^{-1}$.

[89] Thin surface airfall dust layers would not be subject to compaction forces but might instead be "fluffed" by electrostatic forces. Burial depths of greater than several decimeters could create increased packing over a wide areal extent. To affect near-surface vapor fluxes, soils compressed by burial would have to be subsequently exhumed. Widespread exhumation is possible, but the thermophysical and spectral properties of such deposits would be distinct from loose dust. Dispersal in water could also produce lowporosity, low-diffusivity deposits composed of fine dust or coarse/fine mixtures, but the invocation of liquid water as a component in the diagenesis demands additional support from available geologic or geomorphic context data.

[90] The possibility that diffusion coefficients in dustbearing soils could be reduced by a factor of 2 or more as a function of depth may significantly affect models which include interaction with the deep (i.e., $>5 \mathrm{~cm}$ ) regolith.

\subsection{Sand and Dust Mixtures}

[91] Aeolian processes on Mars can produce well-sorted bed forms such as ripples [Sullivan et al., 2005] and largescale features such as dunes [Kieffer et al., 1992]. The Microscopic Imagers on the MER spacecraft have observed soil grains down to the limit of resolution ( $31 \mu \mathrm{m}$ per pixel), and an observable particle size range of $50-200 \mu \mathrm{m}$ has been determined [Herkenhoff et al., 2004; Jerolmack et al., 2006]. Dust particles below the resolution limit are undoubtedly present at the rover sites and are likely to be present in significant quantities at dustier surface locales. The ubiquitous presence of dust on the surface of Mars and observations of dust-rich low thermal inertia regions suggest the strong possibility that mixtures of sand and dust-sized particles occur in some regions. Fluvial processes, volcanic processes, and impacts can agitate and mix surface regolith. If settling times are rapid, the unsorted or poorly sorted character of the bulk material may be retained in the subsequent deposit. Agitation by shifting winds or longterm evolution through thermal expansion and contraction may mix initially separate particle size fractions at the surface in the absence of these more energetic processes.

[92] We have produced and observed mixtures of two types using the 50-80 $\mu \mathrm{m}$ glass beads as the coarse fraction and two types of dust particles. These are not meant to be representative of any particular Mars soil, but instead show the behavior of regolith diffusivity as a function of dust content. Our experiments support the expectation that even in the absence of mobilized and recrystallized salt acting as a pore-filling agent, the diffusive properties of loose surface soils are affected by the presence of pore-filling materials such as dust. Martian fines have a mean particle size of a few microns [Greeley et al., 2000, and references therein], similar to our $1-3 \mu \mathrm{m}$ dust. Crushed JSC Mars-1 dust contains a range of particle sizes from submicron to $0.1 \mathrm{~mm}$. We have focused on homogeneous mixtures of sand and dust-sized particles, though Farmer [1976] suggests that thin dust mantles produced by airfall may fill the interstices of coarser material such that the diffusion coefficient is significantly reduced. Our method includes initial mechanical agitation and no subsequent compaction. The dust contents in samples we have examined range from a clastsupported sediment with a very minor amount of included dust to a matrix-supported, dust-rich deposit containing a minor proportion of separated larger clasts.

[93] The difference in behavior between the two types of mixtures could be very significant for predictions of diffusive behavior of soils and sublimation lags on Mars. The trend seen in Figure 8 indicates that very small particles (1$3 \mu \mathrm{m}$ dust) admixed with coarser grains do little to increase the diffusive resistance to vapor flux if the whole sample is not mechanically compacted. The diffusion coefficient is independent of the mixing fraction up to $X_{f} \sim 0.4$.

[94] If the distribution of finer material includes a broad spectrum of particle sizes (as in crushed JSC Mars-1), i.e., grains roughly equivalent in size to the coarser fraction in addition to fine particulates, then there is a marked reduction in diffusivity as the mass fraction of fines increases. This effect, for uncompacted mixtures, is a reduction in $D$ to $\sim 2.5 \pm 0.3 \mathrm{~cm}^{2} \mathrm{~s}^{-1}$ at $X_{f}=0.4$.

[95] The measured geometric porosity for both types of mixtures increases with $X_{f}$ in a linear fashion as shown in Figure 9a. The porosity of micron-sized dust and beads would reach a value equivalent to pure-dust samples at around $X_{f}=0.6$. The trend with JSC Mars-1 mixtures is shallower, but the ultimate porosity for pure dust is also lower. As we have seen in previous investigations [Hudson et al., 2007], the porosity of dusty soils can be quite large in the absence of mechanical compaction. Even when compressed, the minimum porosity of pure-dust soils is never less than $\sim 80 \%$ in the case of micron-sized dust, and $\sim 60 \%$ in the case of crushed JSC Mars-1. 
[96] As expected for a constant experimental value of $\mathcal{D}_{12}$, the behavior of the obstruction factor follows that of the diffusion coefficient. The increase in overall porosity toward pure dust demands that the degree of convolution of the pore geometry becomes larger to account for the decreasing diffusion coefficient. This is facilitated at lower dust contents in the case of crushed JSC Mars-1 by the broad particle size range. Dust composed only of micronsized particles does not begin to have an appreciable effect on the pore geometry until its mass fraction is substantially higher. However, the implied increase in tortuosity may not occur for some micron-sized dust mixtures since we have seen that the diffusion coefficient of uncompacted pure micron-sized dust is higher than that of any observed mixture. The low bulk density of the dust, the roughly equivalent particle sizes, and the angular particle shapes prevent closer packing and greater degrees of obstruction. Only mechanical compression (or dispersal and settling in a dense lubricating medium such as water) would further reduce the observed $D$, thereby increasing tortuosity.

[97] To determine a tortuosity for each of these simulants and track how their structures change with dust content, many more porosimetry measurements would be needed. Increasing pressure so as to measure the diffusivity in a purely Fickian regime (and thus be able to use equation (3)) becomes difficult as the smallest pore sizes reach the scale of microns. The pore size distribution measurements have indicated that particle size mixtures can either reduce the availability of large pore spaces (i.e., mixture type 1A) or greatly enhance the number and size of the largest pores (i.e., mixture type 2A). For JSC Mars-1 and mixtures of glass beads with fines, the pore distributions were broad. According to the results of Clifford and Hillel [1983, 1986], who show that larger pore spaces accommodate a majority of the flux, even a high proportion of pore spaces experiencing significant numbers of molecule-wall collisions should be less significant to the overall diffusion rate than the few large pathways which may be open in an uncompacted, dust-rich structure. Thus, a real soil may have many pore spaces which experience primarily Knudsen-type diffusion under Mars surface conditions, but a small proportion of large pores undergoing significantly Fickian diffusion may dominate the vapor flux.

[98] Aeolian processes are efficient at sorting particles and can result in particle assemblages which consist mainly of clasts of similar size. Wind ripples such as those in Meridiani Planum show a small-scale variation in the size of surface particles, but the interiors of the ripples are composed of grains of the order of 50-200 $\mu \mathrm{m}$ [Sullivan et al., 2005]. Given the ubiquitous presence of micron-sized dust, and its tendency to adhere to solid surfaces, it is highly likely that some fraction of dust is incorporated into the soil. Dustier locales than Meridiani Planum may exhibit even higher concentrations of fines. However, our results suggest that the diffusive properties of Mars soils will not be greatly affected by the presence of moderate amounts of micronsized dust. Moreover, soils composed primarily of dust may exhibit the highest diffusivities. This conclusion, combined with the large specific surface areas for adsorption and expected large porosities of dusty deposits, supports the notion that dusty regions on Mars are significant compo- nents of past and present-day water cycles and are not isolated from the atmosphere.

[99] The probability that an otherwise homogeneous layer of diffusive material will contain a crack or other efficient path to vapor transport will scale with the area considered. Such pathways may be caused by thermal cycling, microtectonic phenomena, slumping, or (in areas where possible) wetting and drying effects. The samples observed in this controlled laboratory environment are relatively small in size and are not generally subject to phenomena which cause cracking. However, in certain cases where the material exhibited a moderate degree of cohesiveness (e.g., pure dusts or mixtures incorporating crushed JSC Mars-1), cracking was initiated by slumping into the void left by sublimating ice. We did not, however, observe any sudden change in mass loss rate as would be expected if the crack extended from the top surface to the ice.

[100] On Mars, such internal deformations of poor to moderately cohesive regoliths may prevent cracking on the scale of millimeters from affecting the loss rate of ice, which is several $\mathrm{cm}$ beneath the soil surface. If the material were highly cohesive, a crack may penetrate deeply and remain unfilled, resulting in a locally depleted ice layer. The radius of influence of the crack will depend on the mechanical properties of the surrounding soil and whether the crack reaches the ice table.

\subsection{Implications}

[101] The samples we have studied cover a wide range of particle size distributions, porosities, and salt contents. Yet in no case have we observed more than an order of magnitude reduction in fluxes or simulant diffusivity. At most, the diffusivity of our baseline soil simulant (50$80 \mu \mathrm{m}$ glass beads) has been reduced by a factor of about 8 , with more common reductions being a factor of $2-3$. Magnesium sulfate salt produces a moderate reduction at low salt concentrations but has its greatest effect above $10 \mathrm{wt} \%$. Mixtures of coarse and fine particles can produce a decreased diffusion coefficient (as for crushed JSC Mars-1) or no observable effect (as for $1-3 \mu \mathrm{m}$ dust). Mechanical packing of pure-dust samples results in a reduction in diffusivity by a factor of 4-8. Overall, regolith materials similar to the simulants we have studied should not be expected to reduce the transport of water vapor by much more than an order of magnitude. The mechanism of formation of a diffusive barrier is significant in determining the degree of restriction.

[102] Dust and larger particles could be incorporated into ice-rich deposits in a variety of ways, and these dirty ices could then produce lags upon ice sublimation. Two examples of sublimation lags can be considered: midlatitude glaciers and polar layered deposits. For midlatitude glaciers to persist under climate conditions similar to the present for time scales comparable to obliquity variations, they would need to be buried beneath a lag of the order of tens of meters or more.

[103] Polar layered terrain is composed of alternating bright and dark layers which are interpreted as relatively dust-poor and dust-rich deposits, respectively. The layering is believed to be tied to climate cycles and sequential eras of precipitation and sublimation. The dust in the layers may either be deposited contemporaneously with the ice or could be emplaced as part of cyclical global dust storms. In both 
cases, the dust which collects to form the lag would not be subject to compaction forces until the next period of ice deposition. Sublimation during an ice-loss phase would build a lag of uncompressed micron-sized dust particles. If such a lag does not become thick enough to exhibit selfcompaction (possibly on the order of a few decimeters), our experiments show that such an uncompressed material would not reduce surface fluxes of water vapor by more than an order of magnitude relative to unprotected ice at the same temperature (see Figure 5). Additionally, the amount of dust deposited with the ice would have to be significant to build up a monolayer of dust after the sublimation of only meters of ice. Recent observations by subsurface radar sounding instruments estimate an impurity content ranging from 2 to $15 \%$ for the north and south polar layered deposits [Picardi et al., 2005; Plaut et al., 2007], consistent with gravity and topography inversions for density [Zuber et al., 2007].

[104] The diffusivity of a given deposit can be estimated from remote observations as well as geologic and geomorphic contextual information. By considering the context as well as remote or in situ observations, three quantities which feed into porosity and tortuosity estimations, the dust content, the degree of compaction, and the grain size distribution, may be constrained. The results obtained may be used along with homogeneous media diffusion quantities in subsequent models of complex regoliths by adding the contributions of layers with relatively higher or lower dust contents in series.

[105] All observations of salt-bearing and salt-encrusted soils on Mars have thus far been made at low-latitude sites where there is no buried ice. As yet, there are no in situ observations of soil mechanical and geometric properties from a site known to overlie subsurface ice. The Phoenix Lander could perform the first such observations. Trenching activities will also reveal the presence of a cohesive surface layer if one exists. Chemical experiments performed by the Microscopy, Electrochemistry, and Conductivity Analyzer instrument's wet chemistry lab will probe the type and amount of soluble salt component in the soil, and the contribution of this salt to the diffusive barrier may be interpreted in light of the laboratory experiments here.

\section{Notation}

$D$ concentration diffusion coefficient for porous medium.

$D_{F} \quad$ Fickian diffusion coefficient.

$D_{K} \quad$ Knudsen diffusion coefficient.

$D^{\prime}$ uncorrected "raw" diffusion coefficient.

$\mathcal{D}_{12}$ concentration diffusion coefficient in free gas.

$\mathcal{D}_{T}$ thermodiffusion coefficient in free gas.

$\mathcal{D}_{p}$ barodiffusion coefficient in free gas.

$\bar{D}$ reference diffusivity.

$\bar{d}$ average particle diameter.

$J_{1}$ mass flux of water vapor.

$J_{\text {adv }}$ advective component of $J_{1}$.

$J_{\text {diff }}$ diffusive component of $J_{1}$.

$k$ Boltzmann constant.

$M_{c}$ mass of coarse component in mixture.

$M_{f}$ mass of fine component in mixture.

$M_{T}$ total mass of mixture. $p_{0} \quad$ total pressure, $p_{0}=p_{1}+p_{2}$.

$p_{1}$ partial pressure of water.

$p_{\text {ref }}$ reference pressure.

$p_{\mathrm{sv}}^{\text {liq }}$ saturation vapor pressure over liquid water.

$p_{\mathrm{sv}}^{\text {ice }}$ saturation vapor pressure over ice.

$R$ universal gas constant or correlation coefficient.

$r$ pore or particle radius.

$\bar{r}$ average radius.

$T$ temperature.

$T_{\text {ice }}$ temperature of ice surface.

$T_{\text {air }}$ temperature of chamber air measured at hygrometer.

$V_{T}$ total volume of mixture.

$X_{f}$ mass fraction of dust.

$z$ depth.

$z_{\text {corr }}$ correction term.

$\Delta \rho_{1} \quad$ water vapor density difference.

$\Delta z$ sample thickness.

$\kappa$ intrinsic permeability of a porous medium.

$\lambda_{1}$ mean free path of water vapor.

$\mu$ dynamic viscosity.

$\rho_{0}$ total mass density, $\rho_{0}=\rho_{1}+\rho_{2}$.

$\rho_{1}$ density of water vapor.

$\rho_{1 \mathrm{~A}}$ density of water vapor at ice surface.

$\rho_{1 \mathrm{D}}$ density of water vapor at hygrometer.

$\rho_{\text {ice }}$ density of ice.

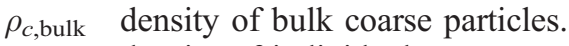

$\rho_{c \text {,true }}$ density of individual coarse particles.

$\rho_{f \text {,bulk }}$ density of bulk fines.

$\rho_{f \text {,true }}$ density of individual fines particles.

$\tau$ tortuosity factor.

$\phi$ porosity.

$\phi_{\text {mix }}$ porosity of mixture.

[106] Acknowledgments. We gratefully acknowledge the assistance of Matt Siegler and Han Man in the preparation and performance of experiments listed here. We wish also to thank Norbert Schorghofer for his advice and scrutiny of early paper drafts. Finally, we are indebted to Barney Farmer for suggesting that experiments using dust mixtures were important in understanding vapor transport on Mars. This work was funded in part by the JPL Director's Discretionary Fund and by NASA's Mars Fundamental Research Program.

\section{References}

Allen, C., R. Morris, D. Lindstrom, M. Lindstrom, and J. Lockwood (1997), JSC Mars-1: Martian regolith simulant, Lunar Planet. Sci., 28, Abstract 1797.

Allen, C., K. Jager, R. Morris, D. Lindstrom, M. Lindstrom, and J. Lockwood (1998), Martian soil simulant available for scientific, educational study, EOS Trans. $A G U, 79(34), 405$.

Boynton, W., et al. (2002), Distribution of hydrogen in the near-surface of Mars: Evidence for subsurface ice deposits, Science, 297, 81-85.

Cabrol, N. A., J. D. Farmer, E. A. Grin, L. Richter, L. Soderblom, R. Li, K. Herkenhoff, G. A. Landis, and R. Arvidson (2006), Aqueous processes at Gusev crater inferred from physical properties of rocks and soils along the Spirit traverse, J. Geophys. Res., 111, E02S20, doi:10.1029/2005JE002490.

Carr, M. (1996), Water on Mars, 229 pp., Oxford Univ. Press, New York.

Chapman, S., and T. Cowling (1970), The Mathematical Theory of NonUniform Gases, 3rd ed., Cambridge Univ. Press, Cambridge, U. K.

Chevrier, V., and P. E. Mathé (2007), Mineralogy and evolution of the surface of Mars: A review, Planet. Space Sci., 55, 289-314.

Clark, B., and D. van Hart (1981), The salts of Mars, Icarus, 45, 370-378, doi:10.1016/0019-1035(81)90041-5.

Clark, B. C., III, A. K. Baird, H. J. Rose Jr., P. Toulmin III, R. P. Christian, W. C. Kelliher, A. J. Castro, C. D. Rowe, K. Keil, and G. R. Huss (1977), The Viking X-ray fluorescence experiment: Analytical methods and early results, J. Geophys. Res., 82, 4577-4594. 
Clifford, S. M., and D. Hillel (1983), The stability of ground ice in the equatorial region of Mars, J. Geophys. Res., 88, 2456-2474.

Clifford, S., and D. Hillel (1986), Knudsen diffusion: The effect of small pore size and low gas pressure on gaseous transport in soil, Soil Sci., 141, $289-297$

Cooper, C., and J. Mustard (2002), Spectroscopy of loose and cemented sulfate-bearing soils: Implications for duricrust on Mars, Icarus, 158 , $42-55$.

Currie, J. (1960), Gaseous diffusion in porous media: Part 1. Dry granular materials, Br. J. Appl. Phys., 11, 318-324.

Farmer, C. (1976), Liquid water on Mars, Icarus, 28, 279-289.

Gille, W., D. Enke, and F. Janowski (2001), Stereological macropore analysis of a controlled pore glass by use of small-angle scattering, J. Porous Mater., 8, 179-191.

Gille, W., D. Enke, and F. Janowski (2002), Pore size distribution and chord length distribution of porous VYCOR glass (PVG), J. Porous Mater., 9 , $221-230$.

Gilmore, M., M. D. Merrill, and R. Castano (2004), Effect of Mars analogue dest deposition on the automated detection of calcite in visible/nearinfrared spectra, Icarus, 172, 641-646.

Greeley, R., R. Leach, B. White, J. Iversen, and J. Pollack (1980), Threshold windspeeds for sand on Mars: Wind tunnel simulations, Geophys. Res. Lett., 7, 121-124.

Greeley, R., G. Wilson, R. Coquilla, B. White, and R. Haberle (2000), Windblown dust on Mars: Laboratory simulations of flux as a function of surface roughness, Planet. Space Sci., 48, 1349-1355.

Gross, F., S. Grek, C. I. Calle, and R. U. Lee (2001), JSC Mars-1 Martian regolith simulant particle charging experiments in a low pressure environment, J. Electrost., 53(4), 257-266.

Herkenhoff, K. E., et al. (2004), Textures of the soils and rocks at Gusev crater from Spirit's Microscopic Imager, Science, 305, doi:10.1126/ science. 3050824

Hoogschagen, J. (1955), Diffusion in porous catalysts and adsorbents, Ind. Eng. Chem., 47(5), 906-912.

Hudson, T., O. Aharonson, N. Schorghofer, C. Farmer, M. Hecht, and N. Bridges (2007), Water vapor diffusion in Mars subsurface environments, J. Geophys. Res., 112, E05016, doi:10.1029/2006JE002815.

Ingersoll, A. P. (1970), Mars: Occurrence of liquid water, Science, 168, 972-973.

Jerolmack, D. J., D. Mohrig, J. P. Grotzinger, D. A. Fike, and W. A. Watters (2006), Spatial grain size sorting in eolian ripples and estimation of wind conditions on planetary surfaces: Application to Meridiani Planum, Mars, J. Geophys. Res., 111, E12S02, doi:10.1029/2005JE002544.

Kieffer, H., , B. Jakosky, C. Snyder, and M. Matthews (Eds.) (1992), Martian aeolian processes, sediments, and features, in Mars, Space Sci. Ser., pp. 730-765, Univ. of Ariz. Press, Tuscon.

Landau, L., and E. Lifshitz (1987), Fluid Mechanics, Pergamon Press, Oxford, U. K

Landis, .A., et al. (2004), Transient liquid water as a mechanism for induration of soil crusts on Mars, Lunar Planet. Sci., 35, Abstract 2188.

Levitz, P. (1993), Knudsen diffusion and excitation transfer in random porous media, J. Phys. Chem., 97, 3813-3818.

Levrard, B., F. Forget, J. Laskar, and F. Montmessin (2005), A GCM recent history of northern martian polar layered deposits: Contribution from past equatorial ice reservoirs, Lunar Planet. Sci., 36, Abstract 1783.

Lide, D. (Ed.) (2003), CRC Handbook of Chemistry and Physics, 84th ed., CRC Press, Boca Raton, Fla.

Malin, M., and K. Edgett (2000), Evidence for recent groundwater seepage and surface runoff on Mars, Science, 288, 2330-2335.

Mason, E., and A. Malinauskas (1983), Gas Transport in Porous Media: The Dusty-Gas Model, Chem. Eng. Monogr., vol. 17, Elsevier, Amsterdam.
Mellon, M., and B. Jakosky (1993), Geographic variations in the thermal and diffusive stability of ground ice on Mars, J. Geophys. Res., 98 $3345-3364$

Mellon, M., and B. Jakosky (1995), The distribution and behavior of Martian ground ice during past and present epochs, J. Geophys. Res., 100, $11,781-11,799$

Mellon, M., B. Jakosky, and S. Postawko (1997), The persistence of equatorial ground ice on Mars, J. Geophys. Res., 102, 19,357-19,369.

Moore, H. J. (1992), Estimates of some physical/mechanical properties of Martian rocks and soillike materials, Open-File Rep. 91-568, U. S. Geol. Surv., Menlo Park, Calif.

Picardi, G., et al. (2005), Radar soundings of the subsurface of Mars, Science, 310, 1925-1928, doi:10.1126/science.1122165.

Plaut, J. J., et al. (2007), Subsurface radar sounding of the south polar layered deposits of Mars, Science, 316, 92-95, doi:10.1126/ science1139672.

Presley, M., and P. Christensen (1989), The distribution and origin of duricrusts on Mars, in Lunar Planet, Sci., 20, 868-869.

Schorghofer, N., and O. Aharonson (2005), Stability and exchange of subsurface ice on Mars, J. Geophys. Res., 110, E05003, doi:10.1029/ 2004JE002350.

Sizemore, H. G., and M. T. Mellon (2007), Laboratory measurements of tortuosity and permeability in Mars analog soils, paper presented at 7 th International Conference on Mars, Lunar and Planet. Inst., Pasadena, Calif., 9-13 July.

Smoluchowski, R. (1968), Mars: Retention of ice, Science, 159, 1348 1350

Sullivan, R., et al. (2005), Aeolian processes at the Mars Exploration Rover Meridiani Planum landing site, Nature, 436, 58-61, doi:10.1038/ nature03641.

Vaniman, D., D. Bish, S. Chipewa, C. Fialips, J. Carrey, and W. Feldman (2004), Magnesium sulfate salts and the history of water on Mars, Nature, $431,663-665$

Wallace, D., and C. Sagan (1979), Evaporation of ice in planetary atmospheres: Ice-covered rivers on Mars, Icarus, 39, 385-400.

Wang, A., et al. (2006), Sulfate deposition in subsurface regolith in Gusev crater, Mars, J. Geophys. Res., 111, (E02S17), doi:10.1029/ 2005JE002513.

Wang, A., J. F. Bell III, and R. Li (2007), Salty soils at Gusev crater as reviealed by Mars Exploration Rover Spirit, Lunar Planet. Sci., 38 , Abstract 1196.

Weitz, C. M., R. C. Anderson, J. F. Bell III, W. H. Farrand, K. E. Herkenhoff, J. R. Johnson, B. L. Jolliff, R. V. Morris, S. W. Squyres, and R. J. Sullivan (2006), Soil grain analyses at Meridiani Planum, Mars, J. Geophys. Res., 111, E12S04, doi:10.1029/2005JE002541.

Yen, A., et al. (2005), An integrated view of the chemistry and mineralogy of martian soils, Nature, 436, 49-54.

Yen, A. S., et al. (2007), Composition and formation of the "Paso Robles" class soils at Gusev crater, Lunar and Planet. Sci., 38, Abstract 2030.

Zalc, J. M., S. C. Reyes, and E. Iglesia (2004), The effects of diffusion mechanism and void structure on transport rates and tortuosity factors in complex porous structures, Chem. Eng. Sci., 59, 2947-2960, doi:10.1016/j.ces.2004.04.028.

Zuber, M. T., R. J. Phillips, J. C. Andrews-Hanna, S. W. Asmar, A. S. Konopliv, F. G. Lemoine, J. J. Plaut, and S. E. Smrekar (2007), Density of mars' south polar layered deposits, Science, 317, 1718-1719, doi:10.1126/science.1146995.

O. Aharonson and T. L. Hudson, Division of Geological and Planetary Sciences, California Institute of Technology, MC 150-21, 1200 East California Avenue, Pasadena, CA 91125, USA. (thudson@gps.caltech.edu) 